AEI-2005-163

DAMTP-2005-81

\title{
State sum construction of two-dimensional open-closed Topological Quantum Field Theories
}

\author{
Aaron D. Lauda ${ }^{1}$ \\ Department of Pure Mathematics and Mathematical Statistics, \\ University of Cambridge, Cambridge CB3 OWB, United Kingdom \\ Hendryk Pfeiffer ${ }^{2}$ \\ Max Planck Institute for Gravitational Physics, \\ Am Mühlenberg 1, 14476 Potsdam, Germany
}

(June 29, 2006)

\begin{abstract}
We present a state sum construction of two-dimensional extended Topological Quantum Field Theories (TQFTs), so-called open-closed TQFTs, which generalizes the state sum of Fukuma-Hosono-Kawai from triangulations of conventional two-dimensional cobordisms to those of open-closed cobordisms, i.e. smooth compact oriented 2-manifolds with corners that have a particular global structure. This construction reveals the topological interpretation of the associative algebra on which the state sum is based, as the vector space that the TQFT assigns to the unit interval. Extending the notion of a two-dimensional TQFT from cobordisms to suitable manifolds with corners therefore makes the relationship between the global description of the TQFT in terms of a functor into the category of vector spaces and the local description in terms of a state sum fully transparent. We also illustrate the state sum construction of an open-closed TQFT with a finite set of D-branes using the example of the groupoid algebra of a finite groupoid.
\end{abstract}

Mathematics Subject Classification (2000): 57R56, 57M99, 81T40, 57Q20.

\section{Introduction}

An $n$-dimensional Topological Quantum Field Theory (TQFT) [1] is a symmetric monoidal functor from the category $\mathbf{n C o b}$ of $n$-dimensional cobordisms to the category Vect $_{k}$ of vector spaces over a given field $k$. The objects of the category $\mathbf{n C o b}$ are smooth compact oriented $(n-1)$-manifolds without boundary, and the morphisms are equivalence classes of smooth compact oriented cobordisms between these, modulo diffeomorphisms that restrict to the identity on the boundary. An $n$-dimensional TQFT therefore associates vector spaces with $(n-1)$-manifolds and linear maps with $n$-dimensional cobordisms. Disjoint unions of manifolds correspond to tensor products of vector spaces and linear maps, and gluing cobordisms along their boundaries corresponds to the composition of linear maps. Note that the empty $(n-1)$-manifold plays the role of the unit object for the tensor product and corresponds to the field $k$.

For $n=2$, the category $\mathbf{n C o b}$ is well understood, and so there are strong results about 2dimensional TQFTs. For these classic results, we refer to [2-4] and to the book [5]. It is known, for example, that 2-dimensional TQFTs are characterized by commutative Frobenius algebras. The objects of $\mathbf{2} \mathbf{C o b}$ are compact 1-manifolds without boundary, i.e. disjoint unions of circles

\footnotetext{
${ }^{1}$ email: A.Lauda@dpmms.cam.ac.uk

${ }^{2}$ email: pfeiffer@aei.mpg.de
} 
$S^{1}$. For the morphisms of $\mathbf{2} \mathbf{C o b}$, one has a description in terms of generators and relations. The generators are these cobordisms:

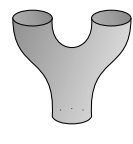

$\mu$

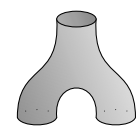

$\Delta$

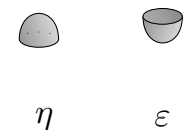

We have drawn them in such a way that their source is at the top and their target at the bottom of the diagram. The TQFT is a functor $Z: \mathbf{2 C o b} \rightarrow \operatorname{Vect}_{k}$. If we denote by $C:=Z\left(S^{1}\right)$ the vector space associated with the circle, the TQFT assigns linear maps $\mu: C \otimes C \rightarrow C, \Delta: C \rightarrow C \otimes C$, $\eta: k \rightarrow C$ and $\varepsilon: C \rightarrow k$ to the morphisms depicted in (1.1). The relations among the morphisms of $\mathbf{2 C o b}$ then imply that $(C, \mu, \eta, \Delta, \varepsilon)$ forms a commutative Frobenius algebra. Conversely, given any commutative Frobenius algebra $C$, there is a functor $Z: \mathbf{2 C o b} \rightarrow \operatorname{Vect}_{k}$ such that $Z\left(S^{1}\right)=C$. We say that the commutative Frobenius algebra provides a global description of the 2-dimensional TQFT. The relevant algebraic structure, namely the commutative Frobenius algebra $(C, \mu, \eta, \Delta, \varepsilon)$, has an immediate topological interpretation in terms of the vector space $C$ associated with the circle, the linear maps $\mu, \eta, \Delta$, and $\varepsilon$ associated with the generators (1.1), and in terms of the relations among the morphisms of $\mathbf{2} \mathbf{C o b}$.

The state sum of Fukuma-Hosono-Kawai [6] forms a different and a priori independent way of defining a 2-dimensional TQFT. This construction starts with a finite-dimensional semisimple algebra $(A, \mu, \eta)$ over a field $k$ of characteristic zero. For every 2-dimensional cobordism $M: \Sigma_{1} \rightarrow$ $\Sigma_{2}$, one considers a triangulation of $M$, and from the data $A, \mu, \eta$, and from the triangulation, one computes the linear map $Z(M): Z\left(\Sigma_{1}\right) \rightarrow Z\left(\Sigma_{2}\right)$ as a state sum. In a state sum, roughly speaking, one colours the simplices of the triangulated manifold $M$ with algebraic data such as the vector space underlying $A$ or the linear maps $\mu, \eta$, and then one 'sums over all colourings' following certain rules. We present this construction in detail in Section 4 below. In particular, one can compute the vector space associated with the circle, and it turns out that this is the centre

$$
Z\left(S^{1}\right)=Z(A)
$$

of the algebra one has started with. The first ' $Z$ ' in (1.2) refers to the functor $Z: \mathbf{2} \mathbf{C o b} \rightarrow$ Vect $_{k}$ whereas the second ' $Z$ ' means centre. The structure of $Z\left(S^{1}\right)$ as a commutative Frobenius algebra can be computed from the algebra $A$, too.

While the centre $Z(A)$ has a clear topological interpretation as outlined above, the algebra $A$ is so far just part of a 'recipe' (the state sum construction), but it is far from obvious whether $A$ itself plays any role in the topology of 2-manifolds.

Given a 2-dimensional TQFT $Z: \mathbf{2 C o b} \rightarrow$ Vect $_{k}$ where $k$ is a field of characteristic zero, one can ask the converse question, namely, whether there is a finite-dimensional semisimple algebra $A$ over $k$ such that one can obtain the given TQFT from the state sum of Fukuma-Hosono-Kawai. Of course, the algebra structure of $A$ needs to be such that $Z(A)=Z\left(S^{1}\right)$, but one also has to understand which Frobenius algebra structure to choose for $A$ in order to recover the appropriate one for $Z(A)$. In order to answer this question, a topological interpretation of the algebra $A$ is clearly desirable.

In the present article, we extend the category $2 \mathrm{Cob}$ from ordinary cobordisms to the category $\mathbf{2} \mathbf{C o b}^{\text {ext }}$ of open-closed cobordisms. These are certain smooth 2-manifolds with corners that can be viewed as cobordisms between compact 1-manifolds with boundary, i.e. between disjoint unions of circles $S^{1}$ and unit intervals $I=[0,1]$. We generalize the notion of a TQFT and the state sum of Fukuma-Hosono-Kawai accordingly, and we show that the algebra $A$ for the state sum construction has a topological interpretation as the vector space associated with the unit interval.

The description of the category $\mathbf{2} \mathbf{C o b}^{\text {ext }}$ in terms of generators and relations goes back to work on boundary conformal field theory by Cardy and Lewellen [7, 8], Lazaroiu [9], and to the work of Moore and Segal [10] and Alexeevski and Natanzon [11]. We have shown the sufficiency of the relations in [12]. In order to get some intuition for the extended cobordism category $\mathbf{2} \mathbf{C o b}^{\text {ext }}$, we 
here display the generators for its morphisms:

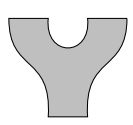

$\mu_{A}$

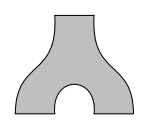

$\Delta_{A}$

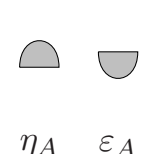

$\eta_{A} \quad \varepsilon_{A}$

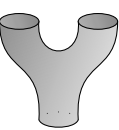

$\mu_{C}$

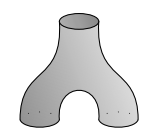

$\Delta_{C}$

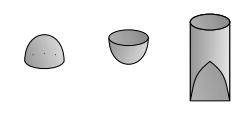

$\eta_{C} \quad \varepsilon_{C} \quad \imath$

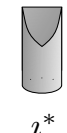

An open-closed TQFT is a symmetric monoidal functor $Z: \mathbf{2} \mathbf{C o b}^{\text {ext }} \rightarrow \mathbf{V e c t}_{k}$. In [12], we have shown that open-closed TQFTs are characterized by what we call knowledgeable Frobenius algebras $\left(A, C, \imath, \imath^{*}\right)$ where the vector space $C:=Z\left(S^{1}\right)$ associated with the circle has the structure of a commutative Frobenius algebra $\left(C, \mu_{C}, \eta_{C}, \Delta_{C}, \varepsilon_{C}\right)$, the vector space $A:=Z(I)$ associated with the interval has the structure of a symmetric Frobenius algebra $\left(A, \mu_{A}, \eta_{A}, \Delta_{A}, \varepsilon_{A}\right)$, and there are linear maps $\imath: C \rightarrow A$ and $\imath^{*}: A \rightarrow C$ subject to certain conditions. For the details, see Section 2.6 below.

In the present article, we show (see Theorem 4.7 below) that for every strongly separable ${ }^{3}$ algebra $A$ over any field $k$ and for every choice of a symmetric Frobenius algebra structure for $A$, there is a knowledgeable Frobenius algebra $\left(A, Z(A), \imath, \imath^{*}\right)$ and a generalization to $\mathbf{2} \mathbf{C o b}^{\text {ext }}$ of the state sum of Fukuma-Hosono-Kawai that yields the open-closed TQFT characterized by $\left(A, Z(A), \imath, \imath^{*}\right)$. Extending the notion of a 2-dimensional TQFT to suitable manifolds with corners therefore reveals which topological role is played by the algebra $A$ that enters the state sum construction.

Why is it important to better understand the role of the algebra A? After all, 2-dimensional TQFTs are well understood, and the state sum of Fukuma-Hosono-Kawai is just one of several ways of finding examples. This question has various answers depending on the view point taken.

Open-closed cobordisms have a natural string theoretic interpretation. Indeed, the generators in (1.3) can be interpreted as the smooth manifolds with corners that underly open and closed string worldsheets. In the state sum, $A$ therefore turns out to be the algebra associated to the open string.

The state sum of Fukuma-Hosono-Kawai is also relevant to recent work on boundary conformal field theory, see, for example $[13,14]$ where the algebra $A$ already appears in connection with the boundary conditions, and so the present article is immediately relevant in this context.

Another reason for better understanding the topological significance of the algebra $A$ is given by attempts to generalize the framework to higher dimensions. For $n \geq 3$, the cobordism category nCob is not fully understood, i.e. $n$-dimensional cobordisms have not been (or even cannot be) classified, and in particular one does not have any description of $\mathbf{n C o b}$ in terms of generators and relations. This makes a full understanding of $n$-dimensional TQFTs much harder if not impossible.

On the other hand, there are some generalizations of the state sum construction of FukumaHosono-Kawai to higher dimensions, notably the 3-dimensional TQFT of Turaev and Viro [15], extended by Barrett and Westbury [16], which produces a 3-dimensional TQFT for any given modular category or, more generally, for suitable spherical categories [17]. The step from dimension 2 to 3, i.e. from the state sum of Fukuma-Hosono-Kawai to that of Turaev-Viro, can be understood as an example of categorification which means replacing algebraic structures based on sets and maps by analogues that are rather based on categories and functors [18]. The dimensional ladder of Crane and Frenkel [19] sketches which sort of algebraic structures one would need in order to

\footnotetext{
${ }^{3}$ It turns out that for a field of arbitrary characteristic, the appropriate class of algebras is that of the strongly separable ones.
} 
construct $n$-dimensional TQFTs from state sums:

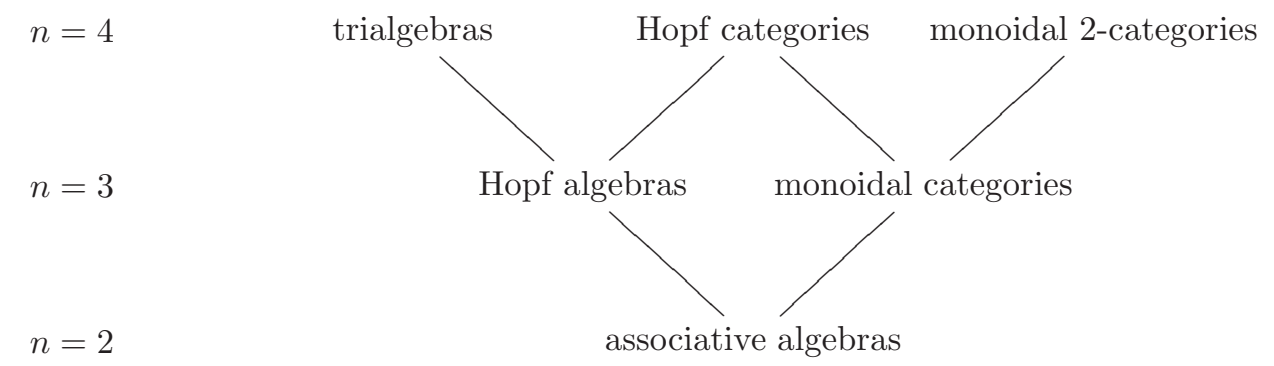

In this diagram, the entry 'associative algebras' refers to the state sum of Fukuma-Hosono-Kawai whereas 'monoidal categories' refers to the Turaev-Viro state sum. For $n=2$, it should actually read 'strongly separable associative algebras'. The appropriate choice of adjectives for the other cases is in fact not systematically understood. In order to settle this question and in order to extend the diagram upwards to higher dimension, one can ask whether it is possible to classify the algebraic structures from which one can construct $n$-dimensional state sum TQFTs for generic $n$.

Whereas the algebraic structures of (1.4) that are relevant to the state sum construction, are closely related to Pachner moves [20] and to the coherence conditions in higher categories, they have no obvious relationship to the global description of the TQFT as a functor $Z: \mathbf{n C o b} \rightarrow \mathbf{V e c t}_{k}$.

By showing that the associative algebra $A$ of the Fukuma-Hosono-Kawai state sum is precisely the vector space $A=Z(I)$ associated with the unit interval in an appropriately extended notion of 2-dimensional TQFT, we have revealed such a relationship for the simplest case $n=2$ of the dimensional ladder (1.4). This raises the question of whether one can find topological interpretations for the other algebraic structures featured in (1.4), presumably by extending the notion of TQFT from conventional cobordisms to manifolds with corners of higher and higher codimension. Further evidence for such a relationship is provided by the Hopf algebra object in 3-dimensional extended TQFTs [21-23] in connection with Kuperberg's 3-manifold invariant which is based on certain Hopf algebras [24].

In the present article, we consider Frobenius algebras not only in the category Vect $_{k}$ of vector spaces, but in any symmetric monoidal Abelian category $\mathcal{C}$. This extends our results without any additional work to Frobenius algebras in the category of graded vector spaces or in the category of chain complexes, etc..

The groupoid algebra $k[\mathcal{G}]$ of a finite groupoid $\mathcal{G}$ forms an example of a strongly separable algebra for suitable fields $k$. We show that our generalized state sum for this algebra yields an easy example of an open-closed TQFT with D-branes.

The present article is structured as follows. In Section 2 we collect the key definitions and facts about symmetric Frobenius algebras, strongly separable symmetric Frobenius algebras, and knowledgeable Frobenius algebras. We also introduce convenient diagrams. In Section 3 we recall the definition of the category $\mathbf{2} \mathbf{C o b}^{\text {ext }}$ of open-closed cobordisms and how to triangulate these. The state sum construction of combinatorial open-closed TQFTs is then presented in Section 4

\section{Frobenius Algebras}

\subsection{Symmetric monoidal categories and string diagrams}

In this section, we review the basics of string diagrams in a symmetric monoidal category. The symmetric monoidal categories of interest will be Abelian symmetric monoidal categories so that the Hom spaces are Abelian groups and the notion of kernels and cokernels are defined. Such categories include the categories of vector spaces, graded vector spaces, $R$-modules for a commutative ring $R$, and chain complexes of each of these structures. We denote a symmetric monoidal category $\mathcal{C}$ as $(\mathcal{C}, \otimes, \mathbb{1}, \alpha, \lambda, \rho, \tau)$ where $\mathcal{C}$ is a category and $\otimes$ provides $\mathcal{C}$ with a monoidal structure with unit object $\mathbb{1}$ whose associator is denoted $\alpha$ and whose left and right unit constraints are given by $\lambda$ and $\rho$. The symmetric braiding is denoted $\tau$. 
We denote the class of objects of a category $\mathcal{C}$ by $|\mathcal{C}|$ and for each object $X \in|\mathcal{C}|$, the identity

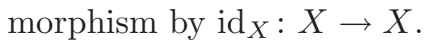

Definition 2.1. Let $(\mathcal{C}, \otimes, \mathbb{1}, \alpha, \lambda, \rho, \tau)$ be a symmetric monoidal category.

1. An object $X$ of $\mathcal{C}$ is called rigid if it has a left-dual $\left(X^{*}, \operatorname{ev}_{X}, \operatorname{coev}_{X}\right)$. This is an object $X^{*}$ of $\mathcal{C}$ with morphisms ev ${ }_{X}: X^{*} \otimes X \rightarrow \mathbb{1}$ (evaluation) and $\operatorname{coev}_{X}: \mathbb{1} \rightarrow X \otimes X^{*}$ (coevaluation) which satisfy the zig-zag identities,

$$
\begin{aligned}
\rho_{X} \circ\left(\operatorname{id}_{X} \otimes \operatorname{ev}_{X}\right) \circ \alpha_{X, X^{*}, X} \circ\left(\operatorname{coev}_{X} \otimes \operatorname{id}_{X}\right) \circ \lambda_{X}^{-1}= & \operatorname{id}_{X} \\
\lambda_{X^{*}} \circ\left(\operatorname{ev}_{X} \otimes \operatorname{id}_{X^{*}}\right) \circ \alpha_{X^{*}, X, X^{*}}^{-1} \circ\left(\operatorname{id}_{X^{*}} \otimes \operatorname{coev}_{X}\right) \circ \rho_{X^{*}}^{-1}= & \operatorname{id}_{X^{*}}
\end{aligned}
$$

2. Let $X$ be a rigid object of $\mathcal{C}$ and $f \in \operatorname{Hom}(X, X)$. The categorical $\operatorname{trace} \operatorname{tr}_{X}(f)$ is defined by,

$$
\operatorname{tr}_{X}(f):=\operatorname{ev}_{X} \circ \tau_{X, X^{*}} \circ\left(f \otimes \operatorname{id}_{X^{*}}\right) \circ \operatorname{coev}_{X} \in \operatorname{Hom}(\mathbb{1}, \mathbb{1}) .
$$

3. The categorical dimension $\operatorname{dim} X$ of a rigid object $X$ of $\mathcal{C}$ is defined by,

$$
\operatorname{dim} X:=\operatorname{tr}_{X}\left(\operatorname{id}_{X}\right) \in \operatorname{Hom}(\mathbb{1}, \mathbb{1}) .
$$

4. For rigid objects $X$ and $Y$ of $\mathcal{C}$ and $f \in \operatorname{Hom}(X, Y)$, the morphism,

$$
\begin{aligned}
f^{*}:= & \lambda_{X^{*}} \circ\left(\operatorname{ev}_{Y} \otimes \operatorname{id}_{X^{*}}\right) \circ\left(\left(\operatorname{id}_{Y^{*}} \otimes f\right) \otimes \operatorname{id}_{X^{*}}\right) \\
& \circ \alpha_{Y^{*}, X, X^{*}}^{-1} \circ\left(\operatorname{id}_{Y^{*}} \otimes \operatorname{coev}_{X}\right) \circ \rho_{Y^{*}}^{-1}: Y^{*} \rightarrow X^{*},
\end{aligned}
$$

is called the dual of $f$.

In the following, we use string diagrams $[25,26]$ to visualize morphisms of a given symmetric monoidal category $\mathcal{C}$ and the identities between them. The diagrams are read from top to bottom. For each object $X \in|\mathcal{C}|$, the identity morphism $\operatorname{id}_{X}$ is denoted by a line labeled ' $X$ ' with an arrow pointing down. The identity morphism $\operatorname{id}_{X^{*}}$ of the dual object has the arrow pointing up. For a morphism $f: X \rightarrow Y$, we write a disc labeled ' $f$ ', called a coupon. This disc has a white side which always faces the reader and a black side which never does so,

$$
\operatorname{id}_{X}=\left.\right|^{X}, \quad \operatorname{id}_{X^{*}}=\left.\right|^{X}, \quad f=\underbrace{\dagger}_{f_{Y}^{f}} .
$$

Composition of morphisms is depicted by vertically concatenating the corresponding diagrams; for example, for morphisms $f: X \rightarrow Y$ and $g: Y \rightarrow Z$,

$$
g \circ f=\overbrace{f_{Y}}^{f_{Y}^{X}}=\overbrace{f_{Z}}^{+X}
$$

The tensor product of morphisms is visualized by putting diagrams next to each other; for example, for $f: X_{1} \rightarrow Y_{1}$ and $g: X_{2} \rightarrow Y_{2}$,

$$
\operatorname{id}_{X_{1} \otimes X_{2}}=f^{X_{1} \otimes X_{2}}=\left.f^{X_{1}}\right|^{X_{2}}, f \otimes g=\underbrace{f X_{1} \otimes X_{2}}_{f Y_{1} \otimes Y_{2}}=\underbrace{f_{g}}_{\left.f_{Y_{1}}\right\}_{Y_{2}}} .
$$


The symmetric braiding is denoted by,

$$
\tau_{X, Y}=\succ^{X Y}
$$

Mac Lane's coherence theorem for monoidal categories [27] then ensures that one can unambiguously translate any such string diagram into a morphism of $\mathcal{C}$. One therefore chooses parentheses for all tensor products that occur in the source and target objects of the morphism and inserts the structure isomorphisms $\alpha, \lambda, \rho$ appropriately. The coherence theorem implies that all possible ways of inserting the structure isomorphisms yield equal morphisms, i.e. that there is a well-defined morphism of $\mathcal{C}$ specified by the diagram. In addition, the coherence theorem allows us to suppress the lines associated with the unit object $\mathbb{1}$.

For a rigid object $X \in \mathcal{C}$, evaluation and coevaluation are represented by these diagrams:

$$
\mathrm{ev}_{X}=\downarrow^{X}, \quad \operatorname{coev}_{X}=\overbrace{X}
$$

The zig-zag identities of (2.1) are represented in string diagrams as:

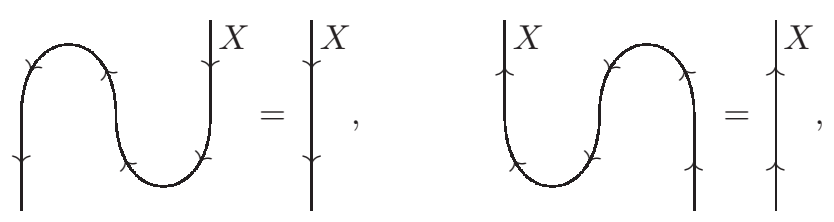

and the definitions of trace (2.2), dimension (2.3) and dual morphism (2.4) are:

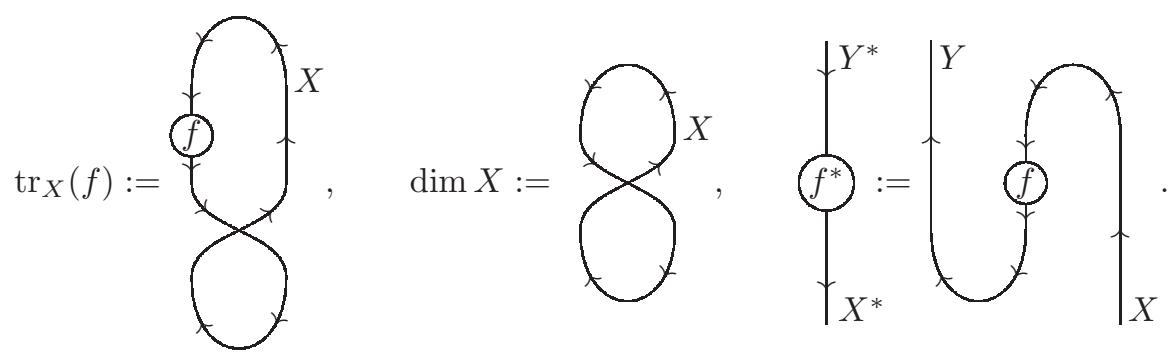

If $\mathcal{C}$ is locally small, the $\operatorname{set} \operatorname{Hom}(\mathbb{1}, \mathbb{1})$ forms a commutative monoid with multiplication $\xi_{1} \cdot \xi_{2}:=$ $\lambda_{\mathbb{1}} \circ\left(\xi_{1} \otimes \xi_{2}\right) \circ \lambda_{\mathbb{1}}^{-1}$ for $\xi_{1}, \xi_{2} \in \operatorname{Hom}(\mathbb{1}, \mathbb{1})$ and unit $\operatorname{id}_{\mathbb{1}}$. The monoid $\operatorname{Hom}(\mathbb{1}, \mathbb{1})$ acts on $\operatorname{Hom}(X, Y)$ for all $X, Y \in|\mathcal{C}|$ by $\xi \cdot f:=\lambda_{Y} \circ(\xi \otimes f) \circ \lambda_{X}^{-1}$ where $f \in \operatorname{Hom}(X, Y)$ and $\xi \in \operatorname{Hom}(\mathbb{1}, \mathbb{1})$.

The coherence theorem now allows us to view the elements of $\operatorname{Hom}(\mathbb{1}, \mathbb{1})$ as scalars by which the entire diagram is multiplied.

\subsection{Frobenius algebras}

We consider Frobenius algebras not only in the symmetric monoidal category Vect $_{k}$ of vector spaces over some fixed field $k$, but in any generic symmetric monoidal category $\mathcal{C}$. The following definitions and results specialize to the usual notions in the case of $\mathcal{C}=$ Vect $_{k}$.

Definition 2.2. Let $(\mathcal{C}, \otimes, \mathbb{1}, \alpha, \lambda, \rho, \tau)$ be a symmetric monoidal category.

1. An algebra object $(A, \mu, \eta)$ in $\mathcal{C}$ consists of an object $A$ and morphisms $\mu: A \otimes A \rightarrow A$ and $\eta: \mathbb{1} \rightarrow A$ of $\mathcal{C}$ such that the following equalities are satisfied:

$$
\mu \circ\left(\operatorname{id}_{A} \otimes \mu\right)=\mu \circ\left(\mu \otimes \operatorname{id}_{A}\right) \circ \alpha_{A, A, A}
$$

and

$$
\begin{aligned}
& \mu \circ\left(\eta \otimes \operatorname{id}_{A}\right)=\lambda_{A}, \\
& \mu \circ\left(\operatorname{id}_{A} \otimes \eta\right)=\rho_{A} .
\end{aligned}
$$


2. A coalgebra object $(A, \Delta, \varepsilon)$ in $\mathcal{C}$ consists of an object $A$ and morphisms $\Delta: A \rightarrow A \otimes A$ and $\varepsilon: A \rightarrow \mathbb{1}$ of $\mathcal{C}$ such that:

$$
\left(\operatorname{id}_{A} \otimes \Delta\right) \circ \Delta=\alpha_{A, A, A} \circ\left(\Delta \otimes \operatorname{id}_{A}\right) \circ \Delta
$$

and

$$
\begin{aligned}
& \left(\varepsilon \otimes \operatorname{id}_{A}\right) \circ \Delta=\lambda_{A}^{-1}, \\
& \left(\operatorname{id}_{A} \otimes \varepsilon\right) \circ \Delta=\rho_{A}^{-1} .
\end{aligned}
$$

3. A Frobenius algebra object $(A, \mu, \eta, \Delta, \varepsilon)$ in $\mathcal{C}$ consists of an object $A$ and of morphisms $\mu$, $\eta, \Delta, \varepsilon$ of $\mathcal{C}$ such that:

(a) $(A, \mu, \eta)$ is an algebra object in $\mathcal{C}$,

(b) $(A, \Delta, \varepsilon)$ is a coalgebra object in $\mathcal{C}$, and

(c) the following compatibility condition, called the Frobenius relation, holds,

$$
\left(\operatorname{id}_{A} \otimes \mu\right) \circ \alpha_{A, A, A} \circ\left(\Delta \otimes \operatorname{id}_{A}\right)=\Delta \circ \mu=\left(\mu \otimes \operatorname{id}_{A}\right) \circ \alpha_{A, A, A}^{-1} \circ\left(\operatorname{id}_{A} \otimes \Delta\right) .
$$

4. A Frobenius algebra object $(A, \mu, \eta, \Delta, \varepsilon)$ in $\mathcal{C}$ is called symmetric if

$$
\varepsilon \circ \mu=\varepsilon \circ \mu \circ \tau \text {. }
$$

It is called commutative if

$$
\mu=\mu \circ \tau
$$

5. Let $\mathcal{C}$ be locally small. A Frobenius algebra object $(A, \mu, \eta, \Delta, \varepsilon)$ in $\mathcal{C}$ is called special (also see $[28])$ if

$$
\varepsilon \circ \eta=\xi_{\mathbb{1}} \cdot \mathrm{id}_{\mathbb{1}} \quad \text { and } \quad \mu \circ \Delta=\xi_{A} \cdot \mathrm{id}_{A} .
$$

for some $\xi_{\mathbb{1}}, \xi_{A} \in \operatorname{Hom}(\mathbb{1}, \mathbb{1})$ that are invertible in the monoid $\operatorname{Hom}(\mathbb{1}, \mathbb{1})$.

The string diagrams for the operations of a Frobenius algebra $(A, \mu, \eta, \Delta, \varepsilon)$ are as follows:
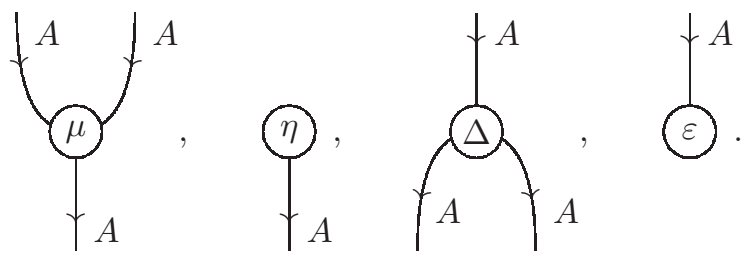

In order to keep the diagrams small, from now on we replace the coupons by vertices and also drop the label ' $A$ ' wherever it is clear from the context:

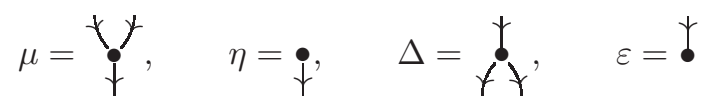

It is understood that the vertices have to be replaced by discs in the paper plane with their white side facing the reader. Furthermore, we drop all labels $\mu, \eta, \Delta$ and $\varepsilon$ where these are evident from the context. For example, we distinguish the operation $\Delta$ from $\mu$ by the arrows of the lines.

The axioms of an algebra and those of a coalgebra then read:
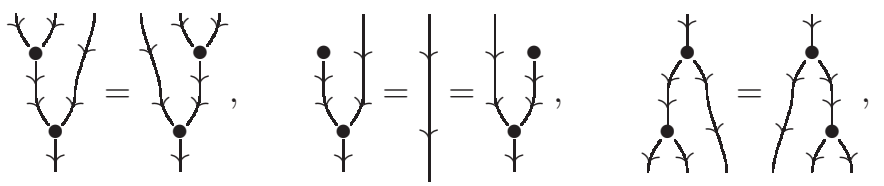

$$
\stackrel{f}{t}=t=f
$$


and the Frobenius relation, commutativity and symmetry are depicted as follows:

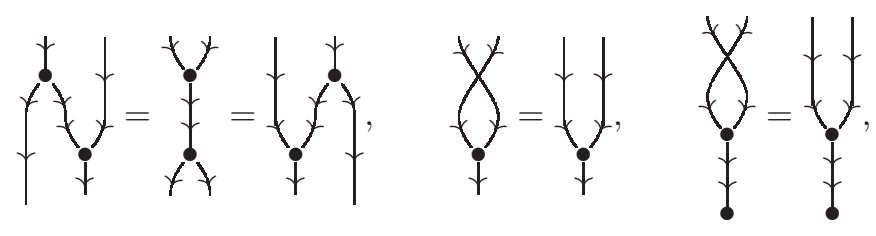

The conditions for a the Frobenius algebra to be special are these:

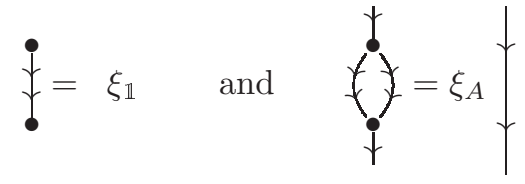

\subsection{Symmetric Frobenius algebras}

In this section, we introduce the notion of a non-degenerate symmetric invariant pairing in order to characterize symmetric Frobenius algebras. In the subsequent sections, we use it to define strongly separable algebras and to classify all symmetric Frobenius algebra structures of a strongly separable algebra.

Definition 2.3. Let $(\mathcal{C}, \otimes, \mathbb{1}, \alpha, \lambda, \rho, \tau)$ be a symmetric monoidal category and $(A, \mu, \eta)$ be an algebra object in $\mathcal{C}$.

1. A pairing on $A$ is a morphism $g: A \otimes A \rightarrow \mathbb{1}$ of $\mathcal{C}$.

2. A pairing $g: A \otimes A \rightarrow \mathbb{1}$ is called non-degenerate if there exists a morphism $g^{*}: \mathbb{1} \rightarrow A \otimes A$ of $\mathcal{C}$ (called the inverse of $g$ ) such that the zig-zag identities hold,

$$
\begin{aligned}
& \rho_{A} \circ\left(\operatorname{id}_{A} \otimes g\right) \circ \alpha_{A, A, A} \circ\left(g^{*} \otimes \operatorname{id}_{A}\right) \circ \lambda_{A}^{-1}=\operatorname{id}_{A}, \\
& \lambda_{A} \circ\left(g \otimes \operatorname{id}_{A}\right) \circ \alpha_{A, A, A}^{-1} \circ\left(\operatorname{id}_{A} \otimes g^{*}\right) \circ \rho_{A}^{-1}=\operatorname{id}_{A} .
\end{aligned}
$$

3. A pairing $g: A \otimes A \rightarrow \mathbb{1}$ is called symmetric if $g=g \circ \tau_{A, A}$.

4. A pairing $g: A \otimes A \rightarrow \mathbb{1}$ is called invariant $t^{4}$ if,

$$
g \circ\left(\operatorname{id}_{A} \otimes \mu\right) \circ \alpha_{A, A, A}=g \circ\left(\mu \otimes \operatorname{id}_{A}\right) .
$$

The string diagrams for a pairing $g: A \otimes A \rightarrow \mathbb{1}$ on an algebra object $(A, \mu, \eta)$ in some symmetric monoidal category $\mathcal{C}$ are as follows:
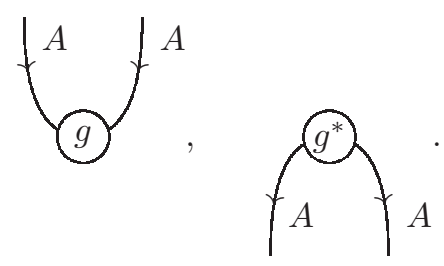

Our shorthand notation using blackboard framing then reads:

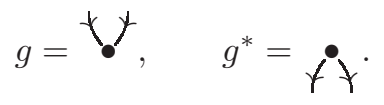

\footnotetext{
${ }^{4}$ Some authors use the term associative rather than invariant, see, for example [5].
} 
The conditions of non-degeneracy, symmetry and invariance are depicted as follows:

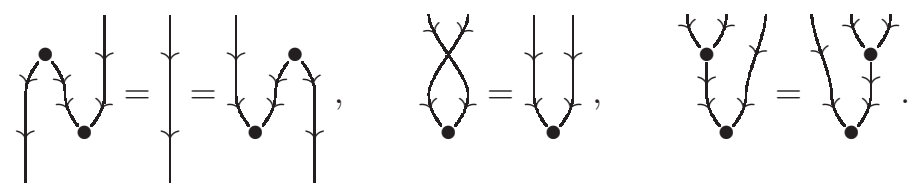

We also use the following shorthand notation for the 'trilinear form' $g^{(3)}:(A \otimes A) \otimes A \rightarrow \mathbb{1}$ which is defined by:

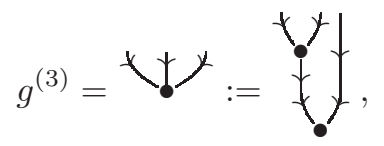

and which has the following cyclic symmetry:

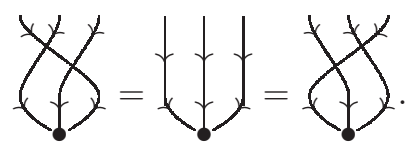

Lemma 2.4. Let $(\mathcal{C}, \otimes, \mathbb{1}, \alpha, \lambda, \rho, \tau)$ be a symmetric monoidal category. Every symmetric Frobenius algebra object $(A, \mu, \eta, \Delta, \varepsilon)$ in $\mathcal{C}$ gives rise to a non-degenerate symmetric invariant pairing $g:=\varepsilon \circ \mu$ on $A$ with inverse $g^{*}:=\Delta \circ \eta$. Conversely, given an algebra object $(A, \mu, \eta)$ in $\mathcal{C}$ and a non-degenerate symmetric invariant pairing $g$ on $A$, there is a symmetric Frobenius algebra object $(A, \mu, \eta, \Delta, \eta)$ with $\Delta:=\left(\mu \otimes \operatorname{id}_{A}\right) \circ \alpha_{A, A, A}^{-1} \circ\left(\operatorname{id}_{A} \otimes g^{*}\right) \circ \rho_{A}^{-1}$ and $\varepsilon:=g \circ\left(\operatorname{id}_{A} \otimes \eta\right) \circ \rho_{A}^{-1}$.

The defining equations used in this lemma can be read diagrammatically as:

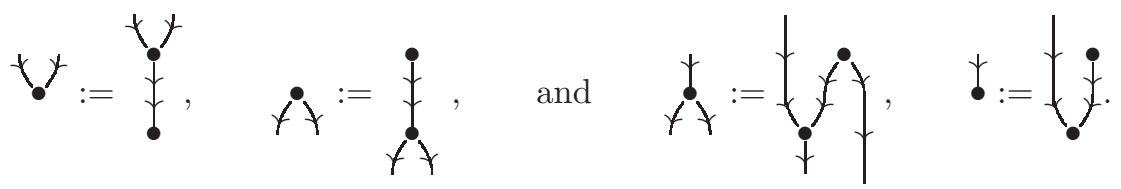

Notice that every symmetric Frobenius algebra object $(A, \mu, \eta, \Delta, \varepsilon)$ in $\mathcal{C}$ is a rigid object of $\mathcal{C}$ with left-dual $^{5}(A, \varepsilon \circ \mu, \Delta \circ \eta)$.

\subsection{Strongly separable algebras}

Every rigid algebra object in a symmetric monoidal category is equipped with a canonical pairing. Recall first the special case of $\mathcal{C}=\operatorname{Vect}_{k}$ for an arbitrary field $k$. Let $A$ be a finite-dimensional algebra over $k$ and denote the left-regular representation by $L: A \rightarrow \operatorname{End}_{k}(A), a \mapsto L_{a}$ with $L_{a}: A \rightarrow A, b \mapsto a b$. By associativity, $L_{a b}=L_{a} \circ L_{b}$ for all $a, b \in A$. The trace of the matrices of the left-regular representation equips $A$ with a canonical bilinear form,

$$
g_{\text {can }}: A \otimes A \rightarrow k, \quad a \otimes b \mapsto \operatorname{tr}_{A}\left(L_{a b}\right),
$$

which can be shown to be symmetric and invariant. We are interested in those algebras for which this canonical bilinear form is non-degenerate. These are the strongly separable algebras. Let us first recall the definition.

Definition 2.5. Let $A$ be an algebra over a commutative ring $r$. We denote by $A^{\text {op }}$ the opposite algebra of $A$, by $A^{e}=A \otimes A^{\mathrm{op}}$ its enveloping algebra and by $\mu: A^{e} \rightarrow A, a \otimes b \mapsto a b$ the augmentation mapping. A is called separable if there is an element $e \in A^{e}$ (called a separability idempotent) such that,

1. $(a \otimes 1) e=(1 \otimes a) e$ holds in $A^{e}$ for all $a \in A$.

\footnotetext{
${ }^{5}$ It is right-dual at the same time, but we do not refer to this property in the following.
} 
2. $\mu(e)=1$.

$A$ is called strongly separable if $A$ is separable with a separability idempotent that satisfies $\tau_{A, A}(e)=e$.

Theorem 2.6 (see, for example [29]). Let $A$ be an algebra over any field $k$. Then the following are equivalent:

1. $A$ is finite-dimensional over $k$, and the canonical bilinear form is non-degenerate.

2. $A$ is strongly separable.

Every strongly separable algebra therefore carries a canonical symmetric Frobenius algebra structure by Lemma 2.4 The following definition of a canonical pairing for generic $\mathcal{C}$ reduces to the canonical bilinear form in the case of $\mathcal{C}=\operatorname{Vect}_{k}$.

Proposition 2.7. Let $(\mathcal{C}, \otimes, \mathbb{1}, \alpha, \lambda, \rho, \tau)$ be a symmetric monoidal category and $(A, \mu, \eta)$ be an algebra object in $\mathcal{C}$ such that the object $A$ is rigid with left-dual $\left(A^{*}, \operatorname{ev}_{A}, \operatorname{coev}_{A}\right)$. Then there is a symmetric invariant pairing on $A$ given by,

$$
g_{\mathrm{can}}:=\operatorname{ev}_{A} \circ \tau_{A, A^{*}} \circ\left(\mu \otimes \operatorname{id}_{A^{*}}\right) \circ \alpha_{A, A, A^{*}}^{-1} \circ\left(\mu \otimes \operatorname{coev}_{A}\right) \circ \rho_{A \otimes A}^{-1}=
$$

which we call the canonical pairing.

Definition 2.8. A rigid algebra object in a symmetric monoidal category is called strongly separable if its canonical pairing is non-degenerate.

By Theorem 2.6 this notion of a strongly separable algebra object in some symmetric monoidal category agrees with the usual definition in the case $\mathcal{C}=\operatorname{Vect}_{k}$. We are not aware of any such result for the more general case of modules over a commutative ring. In order to illustrate how strong the condition of strong separability is, we include the following results and examples from $[29,30]$.

Theorem 2.9. Let $A$ be an algebra over some field $k$.

1. If $A$ is strongly separable, then $A$ is finite-dimensional, separable, and semisimple.

2. If $A$ is separable and commutative, then $A$ is strongly separable.

3. If $A$ is finite-dimensional and semisimple and char $k=0$, then $A$ is strongly separable.

4. If $A$ is finite-dimensional and semisimple and $k$ is a perfect field, then $A$ is separable.

Example 2.10. Let $k$ be a field and $G$ be a finite group.

1. If char $k$ does not divide the order of $G$, then the group algebra $k[G]$ is strongly separable.

2. If char $k$ divides the order of $G$, then $k[G]$ is neither semisimple nor separable.

Example 2.11. Let $k$ be a field and $M_{n}(k)$ be the algebra of $(n \times n)$-matrices over $k$.

1. If char $k$ does not divide $n$, then $M_{n}(k)$ is strongly separable.

2. If char $k$ divides $n$, then $M_{n}(k)$ is semisimple and separable, but not strongly separable.

In both examples, the non-degeneracy of the canonical bilinear form is a convenient criterion for strong separability. We explain below why in the Examples 2.10 (2) and 2.11 (2), the state sum construction fails. In particular, for a finite field of non-zero characteristic $p$, the original Fukuma-Hosono-Kawai state sum [6] cannot be applied to the $(p \times p)$-matrix algebra $A:=M_{p}(k)$ although $k$ is perfect and $M_{p}(k)$ is finite-dimensional, separable, and semisimple. 


\subsection{Strongly separable symmetric Frobenius algebras}

In this section, we compare the pairing $\varepsilon \circ \mu$ of a generic symmetric Frobenius algebra with the canonical pairing. They differ by multiplication with a central element which we call the window element ${ }^{6}$.

In a generic locally small symmetric monoidal category $(\mathcal{C}, \otimes, \mathbb{1}, \alpha, \lambda, \rho, \tau)$, we use the terminology element of $A$ for a morphism $a: \mathbb{1} \rightarrow A$. The set $\operatorname{Hom}(\mathbb{1}, A)$ of elements of an algebra object $(A, \mu, \eta)$ in $\mathcal{C}$ forms a monoid with respect to convolution $a \cdot b:=\mu \circ(a \otimes b) \circ \lambda_{\mathbb{1}}^{-1}$ for elements $a, b \in \operatorname{Hom}(\mathbb{1}, A)$ and with unit $\eta$. An element $a \in \operatorname{Hom}(\mathbb{1}, A)$ is called central if it is contained in the commutative submonoid,

$$
\mathcal{Z}(A):=\left\{a \in \operatorname{Hom}(\mathbb{1}, A): \quad \mu \circ\left(a \otimes \operatorname{id}_{A}\right) \circ \lambda_{A}^{-1}=\mu \circ\left(\operatorname{id}_{A} \otimes a\right) \circ \rho_{A}^{-1}\right\} .
$$

The set of invertible elements of $A$ forms a group $\operatorname{Hom}(\mathbb{1}, A)^{\times} \subseteq \operatorname{Hom}(\mathbb{1}, A)$, and the set of invertible central elements $\mathcal{Z}(A)^{\times}:=\mathcal{Z}(A) \cap \operatorname{Hom}(\mathbb{1}, A)^{\times} \leq \operatorname{Hom}(\mathbb{1}, A)^{\times}$a subgroup. This means in particular that the inverse of every central element is central, too. $\mathcal{Z}(A)$ acts on $\operatorname{Hom}(A, A)$ by

$$
\mathcal{Z}(A) \times \operatorname{Hom}(A, A) \rightarrow \operatorname{Hom}(A, A), \quad(a, f) \mapsto a \cdot f:=\mu \circ(a \otimes f) \circ \lambda_{A}^{-1} .
$$

We also have $\left(a \cdot \operatorname{id}_{A}\right) \circ \eta=a$ and $\left(a \cdot \operatorname{id}_{A}\right) \circ\left(b \cdot \operatorname{id}_{A}\right)=(a \cdot b) \cdot \operatorname{id}_{A}$ for all $a, b \in \mathcal{Z}(A)$ as well as

$$
\mu \circ\left(\left(a \cdot \operatorname{id}_{A}\right) \otimes \operatorname{id}_{A}\right)=\left(a \cdot \operatorname{id}_{A}\right) \circ \mu=\mu \circ\left(\operatorname{id}_{A} \otimes\left(a \cdot \operatorname{id}_{A}\right)\right),
$$

and for a Frobenius algebra object $(A, \mu, \eta, \Delta, \varepsilon)$ also,

$$
\left(\left(a \cdot \operatorname{id}_{A}\right) \otimes \operatorname{id}_{A}\right) \circ \Delta=\Delta \circ\left(a \cdot \operatorname{id}_{A}\right)=\left(\operatorname{id}_{A} \otimes\left(a \cdot \operatorname{id}_{A}\right)\right) \circ \Delta .
$$

The following diagrams show an element $a \in \operatorname{Hom}(\mathbb{1}, A)$, the morphism $a \cdot \mathrm{id}_{A}$, and the centrality condition:

$$
a=(a, \quad a \cdot \operatorname{id}_{A}=\underbrace{a t}_{i}, \quad \underset{f}{f}+f_{i}^{+}
$$

Definition 2.12. Let $(A, \mu, \eta, \Delta, \varepsilon)$ be a symmetric Frobenius algebra object in a locally small symmetric monoidal category $\mathcal{C}$. The window element of $A$ is defined by,

$$
a:=\mu \circ \Delta \circ \eta=
$$

The window element is a central element. The comparison between the pairing $\varepsilon \circ \mu$ of a generic symmetric Frobenius algebra and the canonical pairing can be done as follows.

Proposition 2.13. Let $\mathcal{C}$ be a locally small symmetric monoidal category and $(A, \mu, \eta, \Delta, \varepsilon)$ be a symmetric Frobenius algebra object in $\mathcal{C}$. Then the canonical pairing of $A$ is given by,

$$
g_{\text {can }}=\varepsilon \circ\left(a \cdot \operatorname{id}_{A}\right) \circ \mu,
$$

where $a$ denotes the window element.

\footnotetext{
${ }^{6}$ This terminology is inspired by the open-closed cobordism [12] that is associated with this element.
} 
Proof. Notice that $A$ is a rigid object of $\mathcal{C}$, and so it makes sense to study the canonical pairing (2.36). We use the diagrams of (2.30) for $g:=\varepsilon \circ \mu$ and $g^{*}:=\Delta \circ \eta$ :

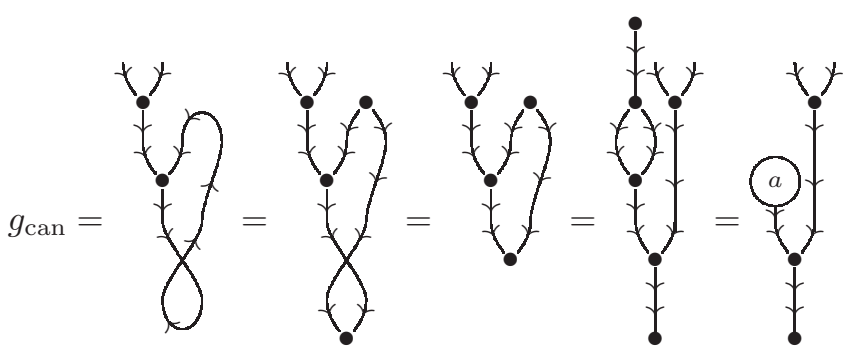

The first equality is the definition; for the second one, we have exploited the fact that $A$ satisfies the zig-zag identities (2.1) both with $\left(A^{*}, \mathrm{ev}_{A}, \operatorname{coev}_{A}\right)$ and with $\left(A, g, g^{*}\right)$; the third equality is symmetry; the fourth one follows from the axioms of a Frobenius algebra; and the fifth is the definition of the window element.

In the above proposition, the pairing $g:=\varepsilon \circ \mu$ is always non-degenerate whereas the canonical pairing is non-degenerate if and only if the algebra is strongly separable.

Theorem 2.14. Let $(\mathcal{C}, \otimes, \mathbb{1}, \alpha, \lambda, \rho, \tau)$ be a locally small symmetric monoidal category and $(A, \mu, \eta, \Delta, \varepsilon)$ be a symmetric Frobenius algebra object in $\mathcal{C}$ with window element $a$. Then the following are equivalent:

1. The algebra object $(A, \mu, \eta)$ is strongly separable.

2. The window element is invertible.

Proof. Let $(A, \mu, \eta)$ be strongly separable. Then the canonical pairing is non-degenerate and therefore has an inverse $g_{\text {can }}^{*}: \mathbb{1} \rightarrow A \otimes A$. We denote the pairing $g:=\varepsilon \circ \mu$ and its inverse $g^{*}:=\Delta \circ \eta$ by the diagrams of (2.30) and the canonical pairing and its inverse by,

$$
g_{\text {can }}=\nvdash_{*}^{\nsucc}, \quad g_{\text {can }}^{*}=\nvdash^{*} \text {. }
$$

Define $\tilde{a}:=\lambda_{A} \circ\left(\varepsilon \otimes \operatorname{id}_{A}\right) \circ g_{\text {can }}^{*}$. Then $\tilde{a}$ is the inverse of $a$ because,

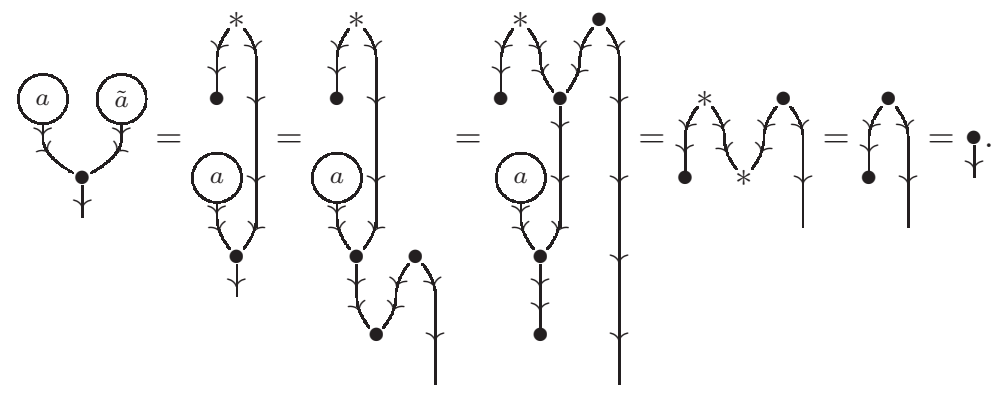

The first equality is the definition of $\tilde{a}$; the second one a zig-zag identity for $\left(A, g, g^{*}\right)$; the third one is a consequence of the axioms of a Frobenius algebra; the fourth one is (2.43); the fifth one is a zig-zag identity for $\left(A, g_{\text {can }}, g_{\text {can }}^{*}\right)$; and the last equation holds by the axioms of a Frobenius algebra.

Conversely, let the window element $a$ have an inverse $a^{-1}$. Then a similar computation shows that the canonical pairing satisfies the zig-zag identities with the inverse $g_{\text {can }}^{*}=\Delta \circ a^{-1}$ and is therefore non-degenerate. 
Combined with Proposition 2.13 the preceding theorem implies that the symmetric Frobenius algebra structures of a given strongly separable algebra are characterized by the invertible central elements. The proposition below describes the extent to which the notion of a strongly separable symmetric Frobenius algebra generalizes the notion of a special symmetric Frobenius algebra.

Proposition 2.15. Let $\mathcal{C}$ be a locally small symmetric monoidal category and $(A, \mu, \eta, \Delta, \varepsilon)$ be a symmetric Frobenius algebra object in $\mathcal{C}$ such that $\operatorname{dim} A$ is invertible in $\operatorname{Hom}(\mathbb{1}, \mathbb{1})$. Then the following are equivalent:

1. The Frobenius algebra object $(A, \mu, \eta, \Delta, \varepsilon)$ is special with $\varepsilon \circ \eta=\xi_{\mathbb{1}} \cdot \operatorname{id}_{\mathbb{1}}$ and $\mu \circ \Delta=\xi_{A} \cdot \mathrm{id}_{A}$ for some invertible $\xi_{\mathbb{1}}, \xi_{A} \in \operatorname{Hom}(\mathbb{1}, \mathbb{1})$.

2. The algebra object $(A, \mu, \eta)$ is strongly separable, and the window element is of the form $a=\zeta \cdot \eta$ for some invertible $\zeta \in \operatorname{Hom}(\mathbb{1}, \mathbb{1})$.

In this case, $\xi_{A}=\zeta$ and $\xi_{\mathbb{1}}=\xi_{A}^{-1} \operatorname{dim} A$.

Proof. If $A$ is special, the window element is $a=\mu \circ \Delta \circ \eta=\left(\xi_{A} \cdot \operatorname{id}_{A}\right) \circ \eta=\xi_{A} \cdot \eta$. It is invertible with $a^{-1}=\xi_{A}^{-1} \cdot \eta$, and so $(A, \mu, \eta)$ is strongly separable.

Conversely, if $(A, \mu, \eta)$ is strongly separable with window element $a=\zeta \cdot \eta$ for some invertible $\zeta \in \operatorname{Hom}(\mathbb{1}, \mathbb{1})$, then the second condition of (2.21) holds with invertible $\xi_{A}=\zeta$. For a symmetric Frobenius algebra object in a symmetric monoidal category, the second condition of (2.21) implies the first one with $\xi_{\mathbb{1}}=\xi_{A}^{-1} \operatorname{dim} A$ :

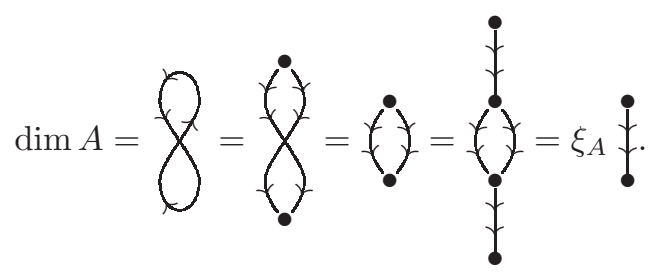

Since $\operatorname{dim} A$ is invertible by assumption, so is $\xi_{\mathbb{1}}$.

Remark 2.16. Given any strongly separable symmetric Frobenius algebra object $(A, \mu, \eta, \Delta, \varepsilon)$ with window element $a$ in a locally small symmetric monoidal category $\mathcal{C}$, the identity

$$
\left(a^{-1} \cdot \operatorname{id}_{A}\right) \circ \mu \circ \Delta=\underbrace{(+)}_{a_{i}^{-1}}=t=\mathrm{id}_{A}
$$

generalizes the 'bubble move' of Fukuma-Hosono-Kawai from the canonical symmetric Frobenius algebra structure to the case of a generic symmetric Frobenius algebra structure. In Section 4 we explain why this generalization is needed in order to obtain a sharp invariant of open-closed cobordisms from the state sum.

For the algebras of Example 2.10(2) and Example 2.112) which are not strongly separable, the morphism $\mu \circ \Delta$ is zero, and so there is no way of obtaining an analogue of the 'bubble move'.

\subsection{Knowledgeable Frobenius algebras}

We have shown in [12] that open-closed TQFTs, i.e. symmetric monoidal functors $Z: \mathbf{2} \mathbf{C o b}^{\text {ext }} \rightarrow \mathcal{C}$ where $\mathcal{C}$ is a symmetric monoidal category, are characterized by knowledgeable Frobenius algebras in $\mathcal{C}$. Here we just recall the definition. For more details, we refer the reader to [12]. 
Definition 2.17. Let $(\mathcal{C}, \otimes, \mathbb{1}, \alpha, \lambda, \rho, \tau)$ be a symmetric monoidal category. A homomorphism of algebras $f: A \rightarrow A^{\prime}$ between two algebra objects $(A, \mu, \eta)$ and $\left(A^{\prime}, \mu^{\prime}, \eta^{\prime}\right)$ in $\mathcal{C}$ is a morphism $f$ of $\mathcal{C}$ such that:

$$
f \circ \mu=\mu^{\prime} \circ(f \otimes f) \quad \text { and } \quad f \circ \eta=\eta^{\prime} .
$$

Definition 2.18. Let $(\mathcal{C}, \otimes, \mathbb{1}, \alpha, \lambda, \rho, \tau)$ be a symmetric monoidal category. A knowledgeable Frobenius algebra $\left(A, C, \imath, \imath^{*}\right)$ in $\mathcal{C}$ consists of,

- a symmetric Frobenius algebra $A=\left(A, \mu_{A}, \eta_{A}, \Delta_{A}, \varepsilon_{A}\right)$,

- a commutative Frobenius algebra $C=\left(C, \mu_{C}, \eta_{C}, \Delta_{C}, \varepsilon_{C}\right)$,

- morphisms $\imath: C \rightarrow A$ and $\imath^{*}: A \rightarrow C$ of $\mathcal{C}$,

such that $\imath: C \rightarrow A$ is a homomorphism of algebra objects in $\mathcal{C}$ and,

$$
\begin{aligned}
\mu_{A} \circ\left(\imath \otimes \mathrm{id}_{A}\right) & =\mu_{A} \circ \tau_{A, A} \circ\left(\imath \otimes \mathrm{id}_{A}\right) & & \text { (knowledge), } \\
\varepsilon_{C} \circ \mu_{C} \circ\left(\operatorname{id}_{C} \otimes \imath^{*}\right) & =\varepsilon_{A} \circ \mu_{A} \circ\left(\imath \otimes \mathrm{id}_{A}\right) & & \text { (duality), } \\
\mu_{A} \circ \tau_{A, A} \circ \Delta_{A} & =\imath \circ \imath^{*} & & \text { (Cardy condition). }
\end{aligned}
$$

Sometimes the folk theorem on the characterization of open-closed TQFTs is stated in such a way that it includes the condition $C=Z(A)$. The following example shows that there exist knowledgeable Frobenius algebras and thereby open-closed TQFTs in which this condition does not hold.

Example 2.19. Let $k$ be a field, char $k \neq 2$ and $n \in \mathbb{N}$ such that char $k$ does not divide $n$. Assume that there exists some $\alpha \in k$ such that $\alpha^{2}=-1 / 2$ (for example $k=\mathbb{C}$ ).

Let $A=M_{n}(k)$ be the $n \times n$-matrix algebra over $k$. Choose a $k$-basis $\left(e_{i j}\right)_{1 \leq i, j \leq n}$ of $A$ such that the multiplication is given by $\mu_{A}\left(e_{i j} \otimes e_{k \ell}\right)=\delta_{j k} e_{i \ell}$ and the unit by $\eta_{A}(1)=\sum_{i=1}^{n} e_{i i}$. The algebra $A$ forms a symmetric Frobenius algebra with $\Delta_{A}\left(e_{i j}\right)=\alpha^{-1} \sum_{k=1}^{n} e_{i k} \otimes e_{k j}$ and $\varepsilon_{A}\left(e_{i j}\right)=\alpha \delta_{i j}$. We compute $\mu_{A} \circ \Delta_{A}=n \alpha^{-1} \cdot \operatorname{id}_{A}$ and the window element $a_{A}=n \alpha^{-1} \cdot \eta_{A}$. It is invertible with $a_{A}^{-1}=n^{-1} \alpha \cdot \eta_{A}$, and so $A$ is strongly separable. In fact, $A$ is special with $\xi_{A}=n \alpha^{-1}$ and $\xi_{\mathbb{1}}=n \alpha$. Obviously, $Z(A) \cong k$.

Let $C=k[X] /\left(X^{2}-1\right)$. A $k$-basis is given by $(1, X)$. $C$ becomes a commutative Frobenius algebra with $\Delta_{C}(1)=1 \otimes X+X \otimes 1, \Delta_{C}(X)=1 \otimes 1+X \otimes X, \varepsilon_{C}(1)=0$, and $\varepsilon_{C}(X)=1$. We compute $\left(\mu_{C} \circ \Delta_{C}\right)(c)=2 X c$ for all $c \in C$, and the window element is $a_{C}=2 X$. It is invertible with $a_{C}^{-1}=X / 2$, and so $C$ is strongly separable, too, but it is not special.

If we define $\imath: C \rightarrow A$ by $\imath(1)=\eta_{A}(1)$ and $\imath(X)=-\eta_{A}(1)$, and $\imath^{*}: A \rightarrow C$ by $\imath^{*}\left(e_{i j}\right)=$ $\delta_{i j} \alpha(X-1)$, then $\left(A, C, \imath, \imath^{*}\right)$ forms a knowledgeable Frobenius algebra. Observe that $Z(A)$ is 1-dimensional over $k$, but $C$ is 2 -dimensional, and so $Z(A) \neq C$.

\subsection{Idempotents}

In this section, we show that every strongly separable symmetric Frobenius algebra $A$ in an Abelian symmetric monoidal category $\mathcal{C}$ gives rise to a knowledgeable Frobenius algebra $\left(A, C, \imath, \imath^{*}\right)$ in $\mathcal{C}$. In $\operatorname{Vect}_{k}, C$ is isomorphic to the centre of $A$. In general, it arises as the image of the following canonical idempotent.

Proposition 2.20. Let $\left(A, \mu_{A}, \eta_{A}, \Delta_{A}, \varepsilon_{A}\right)$ be a strongly separable symmetric Frobenius algebra object in a locally small symmetric monoidal category $\mathcal{C}$ and let $a^{-1}$ denote the inverse of the window element of $A$. Then the morphism

$$
p=(\frac{p}{t}:=\left(a^{-1} \cdot \mathrm{id}_{A}\right) \circ \mu_{A} \circ \tau_{A, A} \circ \Delta_{A}=\underbrace{-1}_{+}
$$


has the following properties,

1. $p^{2}=p$,

2. $p \circ \eta_{A}=\eta_{A}$,

3. $\varepsilon_{A} \circ p=\varepsilon_{A}$,

4. $p \circ \mu_{A} \circ(p \otimes p)=\mu_{A} \circ(p \otimes p)=p \circ \mu_{A} \circ\left(p \otimes \operatorname{id}_{A}\right)=p \circ \mu_{A} \circ\left(\operatorname{id}_{A} \otimes p\right)$,

5. $(p \otimes p) \circ \Delta_{A} \circ p=(p \otimes p) \circ \Delta_{A}=\left(p \otimes \operatorname{id}_{A}\right) \circ \Delta_{A} \circ p=\left(\operatorname{id}_{A} \otimes p\right) \circ \Delta_{A} \circ p$,

6. $c=p \circ c$ for all $c \in \mathcal{Z}(A)$,

7. $\left(c \cdot \operatorname{id}_{A}\right) \circ p=p \circ\left(c \cdot \operatorname{id}_{A}\right)$ for all $c \in \mathcal{Z}(A)$,

8. $\mu_{A} \circ\left(p \otimes \operatorname{id}_{A}\right)=\mu_{A} \circ \tau_{A, A} \circ\left(p \otimes \operatorname{id}_{A}\right)$.

In $\operatorname{Vect}_{k}$, condition (1) states that $p$ is a projector; condition (8) says that its image is contained in the centre $Z(A)$, and condition (6) says that the centre $Z(A)$ is contained in the image of $p$, and so $p$ projects onto the centre $Z(A)$. Whereas this $Z(A)$ arises as a subspace $Z(A)=\operatorname{im} p \subseteq A$, the centre $\mathcal{Z}(A)$ according to (2.37) consists of morphisms $\mathbb{1} \rightarrow A$. In Vect $_{k}$, one can evaluate any such morphism $a \in \mathcal{Z}(A)$ at the unit $1 \in k$ of the field and finds that $a(1) \in Z(A) \subseteq A$.

Note that the idempotent (2.53) is precisely $p=\mu_{A} \circ \tau_{A, A} \circ \Delta_{A}^{\text {(can) }}$ where $\Delta_{A}^{\text {(can) }}$ refers to the canonical symmetric Frobenius algebra structure on $A$.

In the state sum, the idempotent (2.53) appears whenever a unit interval is closed to a circle, i.e. it is closely related with the generators $\imath$ and $\imath^{*}$ of (1.3). The image of an idempotent can be defined in any Abelian category as follows.

Proposition 2.21 (see, for example [31]). Let $\mathcal{C}$ be an Abelian category and $p: A \rightarrow A$ be an idempotent. The image factorization of $p$ yields an object $p(A)$, called the image of $p$, which is unique up to isomorphism, together with morphisms coim $p: A \rightarrow p(A)$ (called the co-image) and $\operatorname{im} p: p(A) \rightarrow A$ (called the image) such that the following diagram commutes:

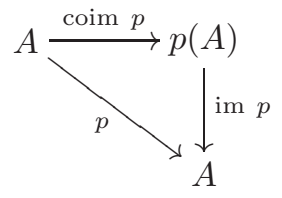

Since $\mathcal{C}$ is Abelian, the idempotent $p$ is split. The splitting is given precisely by the two morphisms of the image factorization, and so we have $\operatorname{id}_{p(A)}=\operatorname{coim} p \circ \operatorname{im} p$. Therefore, the short exact sequence

$$
0 \longrightarrow N_{p} \stackrel{\operatorname{ker} p}{\longrightarrow} A \underset{\operatorname{im} p}{\stackrel{\operatorname{coim} p}{\rightleftarrows}} p(A) \longrightarrow 0
$$

is split as indicated. Here $N_{p}$ denotes the kernel of $p$. This determines the structure of $A \cong$ $N_{p} \oplus p(A)$ in terms of the following biproduct:

$$
N_{p} \underset{\text { coker } p}{\stackrel{\operatorname{ker} p}{\rightleftarrows}} N_{p} \oplus p(A) \underset{\operatorname{im} p}{\stackrel{\operatorname{coim} p}{\rightleftarrows}} p(A) .
$$

The sequence from right to left is split exact, too.

Theorem 2.22. Let $\mathcal{C}$ be an Abelian symmetric monoidal category and $(A, \mu, \eta, \Delta, \varepsilon)$ be a strongly separable symmetric Frobenius algebra object in $\mathcal{C}$ with window element $a$. Then there exists a knowledgeable Frobenius algebra $\left(A, C, \imath, \imath^{*}\right)$ where $C=p(A)$ is the image of the idempotent (2.53), 
$\imath=\operatorname{im} p$, and $\imath^{*}=\operatorname{coim} p \circ\left(a \cdot \operatorname{id}_{A}\right)$. The commutative Frobenius algebra structure of $C$ is given by,

$$
\begin{aligned}
\mu_{C} & =\operatorname{coim} p \circ \mu_{A} \circ(\operatorname{im} p \otimes \operatorname{im} p), \\
\eta_{C} & =\operatorname{coim} p \circ \eta_{A}, \\
\Delta_{C} & =(\operatorname{coim} p \otimes \operatorname{coim} p) \circ \Delta_{A} \circ\left(a \cdot \operatorname{id}_{A}\right) \circ \operatorname{im} p, \\
\varepsilon_{C} & =\varepsilon_{A} \circ\left(a^{-1} \cdot \operatorname{id}_{A}\right) \circ \operatorname{im} p .
\end{aligned}
$$

Proof. The proof uses Proposition 2.20] and Proposition 2.21]

We show below in Section 4 that this knowledgeable Frobenius algebra is precisely the one that is obtained from our generalized state sum for the strongly separable algebra $A$. The following proposition introduces two families of morphisms that are needed in order to show that the morphisms associated with triangulated open-closed cobordisms do not depend on the triangulation of the boundary.

Let $(A, \mu, \eta, \Delta, \varepsilon)$ be a Frobenius algebra object in a locally small symmetric monoidal category $\mathcal{C}$. For $k \in \mathbb{N}$, we denote by

$$
\mu^{(k+1)}:=\mu \circ\left(\mu^{(k)} \otimes \operatorname{id}_{A}\right), \quad \mu^{(2)}:=\mu, \quad \mu^{(1)}:=\operatorname{id}_{A}
$$

and by

$$
\Delta^{(k+1)}:=\left(\Delta^{(k)} \otimes \operatorname{id}_{A}\right) \circ \Delta, \quad \Delta^{(2)}:=\Delta, \quad \Delta^{(1)}:=\operatorname{id}_{A}
$$

the iterated multiplication and comultiplication. We also write $A^{\otimes(k+1)}:=A^{\otimes k} \otimes A, A^{\otimes 1}:=A$ and $A^{\otimes 0}:=\mathbb{1}$, and for $a \in \mathcal{Z}(A), a^{k+1} \cdot \operatorname{id}_{A}:=\left(a^{k} \cdot \operatorname{id}_{A}\right) \circ\left(a \cdot \operatorname{id}_{A}\right)$ and $a^{0} \cdot \operatorname{id}_{A}:=\operatorname{id}_{A}$.

Proposition 2.23. Let $\mathcal{C}$ be a locally small symmetric monoidal category and $(A, \mu, \eta, \Delta, \varepsilon)$ be a strongly separable symmetric Frobenius algebra object in $\mathcal{C}$ with window element $a$. Then for $k, \ell \in \mathbb{N}$, the morphisms

$$
\begin{aligned}
P_{k \ell} & :=\Delta^{(k)} \circ\left(a^{-(k-1)} \cdot \operatorname{id}_{A}\right) \circ \mu^{(\ell)}: A^{\otimes \ell} \rightarrow A^{\otimes k}, \\
Q_{k \ell} & :=\Delta^{(k)} \circ\left(a^{-(k-1)} \cdot \operatorname{id}_{A}\right) \circ p \circ \mu^{(\ell)}: A^{\otimes \ell} \rightarrow A^{\otimes k},
\end{aligned}
$$

satisfy

$$
P_{k \ell} \circ P_{\ell m}=P_{k m} \quad \text { and } \quad Q_{k \ell} \circ Q_{\ell m}=Q_{k m}
$$

for all $k, \ell, m \in \mathbb{N}$. Here $p$ denotes the idempotent of (2.53). In particular, $P_{k k}$ and $Q_{k k}$ are idempotents, and we have $P_{11}=\mathrm{id}_{A}$ and $Q_{11}=p$.

Proof. In any symmetric Frobenius algebra, we have

$$
\mu^{(k)} \circ \Delta^{(k)}=a^{(k-1)} \cdot \operatorname{id}_{A},
$$

which implies both claims.

Corollary 2.24. Let $\mathcal{C}$ be an Abelian symmetric monoidal category and $(A, \mu, \eta, \Delta, \varepsilon)$ be a strongly separable symmetric Frobenius algebra object in $\mathcal{C}$. Then there are isomorphisms

$$
P_{k k}\left(A^{\otimes k}\right) \cong A \quad \text { and } \quad Q_{k k}\left(A^{\otimes k}\right) \cong p(A)
$$

for all $k \in \mathbb{N}$.

Proof. The isomorphisms with their inverses are given by

$$
\begin{gathered}
\Phi_{k}=\operatorname{coim} P_{k k} \circ P_{k 1}: A \rightarrow P_{k k}\left(A^{\otimes k}\right), \\
\Phi_{k}^{-1}=P_{1 k} \circ \operatorname{im~} P_{k k}: P_{k k}\left(A^{\otimes k}\right) \rightarrow A
\end{gathered}
$$

as well as

$$
\begin{aligned}
\Psi_{k} & =\operatorname{coim} Q_{k k} \circ Q_{k 1} \circ \operatorname{im} p: p(A) \rightarrow Q_{k k}\left(A^{\otimes k}\right), \\
\Psi_{k}^{-1} & =\operatorname{coim} p \circ Q_{1 k} \circ \operatorname{im~} Q_{k k}: Q_{k k}\left(A^{\otimes k}\right) \rightarrow p(A) .
\end{aligned}
$$




\section{Open-closed cobordisms}

\subsection{Smooth open-closed cobordisms}

In this section, we briefly review the definition of the category $\mathbf{2} \mathbf{C o b}^{\text {ext }}$ of open-closed cobordisms. These are smooth 2-manifolds with corners that have a particular global structure as follows.

Recall that a smooth $k$-manifold with corners $M$ is a topological $k$-manifold such that every point has a neighbourhood homeomorphic to an open subset of $\mathbb{R}_{+}^{k}:=[0, \infty)^{k}$. The transition functions are required to be the restrictions to $\mathbb{R}_{+}^{k}$ of diffeomorphisms between open subsets of $\mathbb{R}^{k}$.

For each $p \in M$, we define $c(p) \in \mathbb{N}_{0}$ to be the number of zero coefficients in local coordinates $\varphi(p) \in \mathbb{R}_{+}^{k}$. The result is independent of the chosen coordinate system. A connected face of $M$ is the closure of a component of $\{p \in M: c(p)=1\}$. A face is a free union of pairwise disjoint connected faces. A $k$-dimensional manifold with faces is a smooth $k$-manifold with corners such that every $p \in M$ is contained in $c(p)$ different connected faces.

A $k$-dimensional $\langle 2\rangle$-manifold $M$ is a $k$-dimensional manifold with faces with a specified pair $\left(\partial_{0} M, \partial_{1} M\right)$ of faces of $M$ such that $\partial_{0} M \cup \partial_{1} M=\partial M$ (the boundary of $M$ as a topological manifold) and such that $\partial_{0} M \cap \partial_{1} M$ is a face of both $\partial_{0} M$ and $\partial_{1} M$. A diffeomorphism $f: M \rightarrow N$ between $\langle 2\rangle$-manifolds $M$ and $N$ is a diffeomorphism of the underlying manifolds with corners that satisfies $f\left(\partial_{0} M\right)=\partial_{0} N$ and $f\left(\partial_{1} M\right)=\partial_{1} N$.

In the following, we are interested in 2-dimensional $\langle 2\rangle$-manifolds. The following is a typical example:

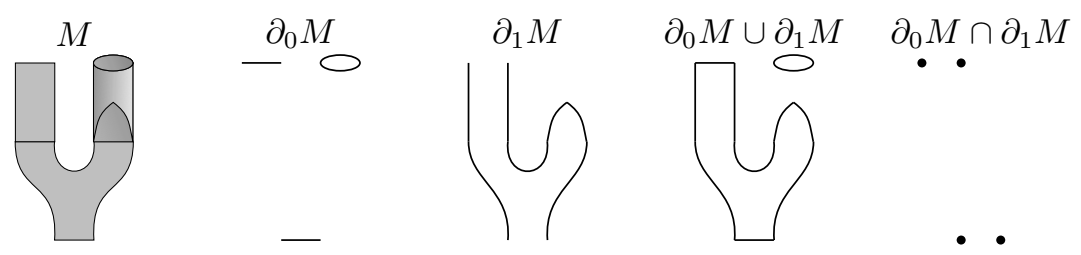

An open-closed cobordism is a compact oriented 2-dimensional $\langle 2\rangle$-manifold $M$ whose distinguished faces we denote by $\left(\partial_{0} M, \partial_{1} M\right)$. We call $\partial_{0} M$ the black boundary and $\partial_{1} M$ the coloured boundary. Two open-closed cobordisms are considered equivalent if there is an orientation preserving diffeomorphism of $\langle 2\rangle$-manifolds that restricts to the identity on the black boundary.

The black boundary $\partial_{0} M$ of an open-closed cobordism is diffeomorphic to a free union of circles $S^{1}$ and unit intervals $I=[0,1]$. One can glue open-closed cobordisms along the components of their black boundary just as one intuitively expects and as it is indicated by the pictures above. For the details, we refer to Section 3 of [12]. There we have defined the symmetric monoidal category $\mathbf{2} \mathbf{C o b}^{\text {ext }}$ as the skeleton of this extended cobordism category. Its objects are finite sequences $\vec{n}=\left(n_{1}, \cdots, n_{k}\right)$ with $n_{i} \in\{0,1\}$ for all $i$ which define smooth oriented compact 2-manifolds $C_{\vec{n}}$ that form the disjoint union of a unit interval for all $n_{i}=1$ and of a circle for all $n_{i}=0$. The morphisms of $\mathbf{2} \mathbf{C o b}^{\text {ext }}$ are equivalence classes of open-closed cobordisms between these $C_{\vec{n}}$. The tensor product is the free union of manifolds, i.e. juxtaposition of the corresponding diagrams, and the composition of morphisms is the gluing of the open-closed cobordisms along their black boundaries. The identity morphisms are cylinders over the compact oriented 1-manifolds that represent the objects.

For the purpose of the present work, it is sufficient to keep in mind that any morphism of the category $\mathbf{2} \mathbf{C o b}{ }^{\text {ext }}$ can be obtained from a finite number of copies of the generators (1.3) by taking tensor products and by taking compositions.

We have also shown in [12] that any two equivalent open-closed cobordisms are related by a finite sequence of moves. These moves are precisely the defining equations of a knowledgeable Frobenius algebra $\left(A, C, \imath, \imath^{*}\right)$ when the operations $\mu_{A}, \Delta_{A}, \ldots, \imath^{*}$ are replaced by the morphisms depicted in (1.3). For example, to the associative law in the symmetric Frobenius algebra 
$\left(A, \mu_{A}, \eta_{A}, \Delta_{A}, \varepsilon_{A}\right)$, there corresponds the following move:

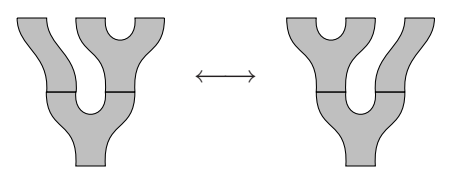

We can summarize the results of [12] on the structure of the category $\mathbf{2} \mathbf{C o b}^{\text {ext }}$ as follows.

Theorem 3.1. The category $\mathbf{2} \mathbf{C o b}^{\text {ext }}$ of open-closed cobordisms is the strict symmetric monoidal category freely generated by a knowledgeable Frobenius algebra object $\left(A, C, \imath, \imath^{*}\right)$. This generating knowledgeable Frobenius algebra object consists of the diffeomorphism type $\vec{n}=(1)$ of the unit interval $A=I$ which forms a symmetric Frobenius algebra $\left(A, \mu_{A}, \eta_{A}, \Delta_{A}, \varepsilon_{A}\right)$; the diffeomorphism type $\vec{n}=(0)$ of the circle $C=S^{1}$ which forms a commutative Frobenius algebra $\left(C, \mu_{C}, \eta_{C}, \Delta_{C}, \varepsilon_{C}\right)$; together with the morphisms $\imath$ and $\imath^{*}$. The morphisms $\mu_{A}, \eta_{A}, \ldots, \imath^{*}$ are precisely the equivalence classes of the open-closed cobordisms depicted in (1.3).

\subsection{Combinatorial open-closed cobordisms}

Open-closed cobordisms can be triangulated as follows. We use the terminology of [32].

Given an open-closed cobordism $M$, the underlying topological manifold is a compact oriented 2-manifold with boundary. We therefore have a finite simplicial complex $K$ whose underlying polyhedron we denote by $|K| \subseteq \mathbb{R}^{p}$ for some $p$, and a homeomorphism $T_{M}:|K| \rightarrow M$ which we call a triangulation. The simplicial complex $K$ satisfies the conditions that guarantee that $|K|$ forms an oriented topological 2-manifold, i.e. the link of each $d$-simplex is a $(1-d)$-sphere iff the simplex is in the interior of $|K|$, and it is a $(1-d)$-ball iff the simplex is in the boundary of $|K|$. Furthermore, for each 2-simplex $\sigma$, it is specified whether $\sigma$ or its opposite oriented simplex $\sigma^{*}$ is contained in $|K|$, and each 1-simplex in the interior of $|K|$ appears as a face of precisely two 2 -simplices with opposite induced orientations.

If $M$ and $N$ are equivalent open-closed cobordisms, their underlying topological manifolds are homeomorphic. If we have triangulations $T_{M}:|K| \rightarrow M$ and $\tilde{T}_{M}:|L| \rightarrow N$ with simplicial complexes $K$ and $L$, Pachner's theorem [20] says that the simplicial complexes $K$ and $L$ are related by a finite sequence of moves. These moves are the bistellar moves (called the 1-3 and 2-2 move),
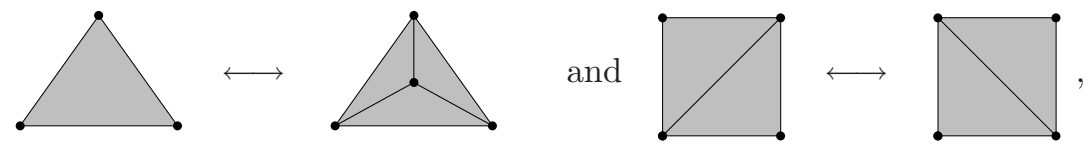

applicable to all 2-simplices, and the elementary shellings
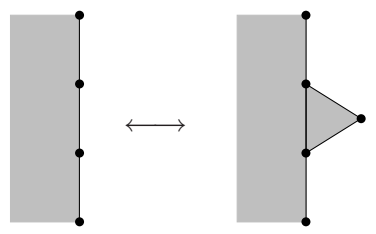

and

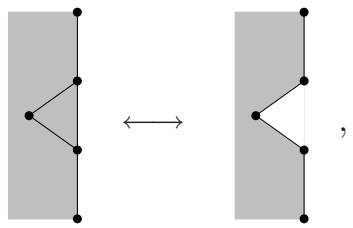

applicable to certain 2-simplices some of whose faces coincide with the boundary. The interior of the manifold is indicated by the shading in our pictures. Recall that for finite simplicial complexes which represent compact manifolds with non-empty boundary, each bistellar move can be obtained from a finite sequence of elementary shellings.

The set of corners $\partial_{0} M \cap \partial_{1} M$ of every open-closed cobordism $M$ is a finite set. Given some triangulation $T_{M}:|K| \rightarrow M$, we can apply a finite sequence of elementary shellings in order to subdivide the 1-simplices in the boundary in such a way that to every corner of $M$, there corresponds a 0 -simplex in $K$, i.e. that $\partial_{0} M \cap \partial_{1} M \subseteq T_{M}\left(\left|K_{0}\right|\right)$ where $K_{0} \subseteq K$ denotes the 0 -skeleton of $K$. From now on we assume, without loss of generality, that every triangulation has 
this property. Given a 1-simplex $\sigma \in K$ in the boundary, we therefore have either $T_{M}(|\sigma|) \subseteq \partial_{0} M$ or $T_{M}(|\sigma|) \subseteq \partial_{1} M$, i.e. the 1-simplices in the boundary are either black or coloured.

Both elementary shellings of (3.4) replace two boundary 1-simplices (edges) by a single edge or vice versa. For triangulations with the special property, each of the elementary shellings (3.4) belongs to one of the following four types:

1. two black edges $\longleftrightarrow$ one black edge,

2. two coloured edges $\longleftrightarrow$ one coloured edge,

3. one black and one coloured edge $\longleftrightarrow$ one black edge,

4. one black and one coloured edge $\longleftrightarrow$ one coloured edge.

It is not difficult to see that the elementary shellings of type (3.) and (4.) can be obtained from a finite sequence of bistellar moves and elementary shellings of type (1.) and (2.).

When we construct open-closed TQFTs in Section 4 below, we consider triangulations of the open-closed cobordisms and then show that the linear map associated with every given cobordism is invariant under the bistellar moves (3.3.3) and under elementary shellings of type (1.) and (2.). Then this linear map is independent of the choice of the triangulation.

\subsection{Smoothing theory}

When one studies smooth manifolds by combinatorial techniques, the relation between combinatorial and smooth manifolds is described by two types of theorems:

- Triangulation: Every compact smooth manifold (with boundary) admits a Whitehead triangulation. If two such manifolds are diffeomorphic, then their triangulations are related by a finite sequence of the appropriate Pachner moves.

- Smoothing: Given a finite simplicial complex $K$ that satisfies the conditions which ensure that its underlying polyhedron $|K|$ forms a topological manifold (with boundary), one needs to know (a) under which conditions there exists a smooth manifold that has $|K|$ as its triangulation and (b) whether the resulting smooth manifold is unique up to diffeomorphism.

Such theorems are available in order to compare smooth manifolds with boundary and combinatorial manifolds with boundary, but we are not aware of any systematic treatment for manifolds with corners, manifolds with faces, or $\langle 2\rangle$-manifolds.

In the preceding section, we have solved the triangulation problem for open-closed cobordisms by resorting to the underlying topological manifold which is just a topological 2-manifold with boundary. It admits a triangulation, and this triangulation is unique up to combinatorial equivalence, i.e. Pachner moves, by the validity of the Combinatorial Triangulation Conjecture and the Hauptvermutung for 2-dimensional manifolds, see, for example [33]. We have then dealt with the corner points 'by hand'.

The other direction, a solution to the smoothing problem, is not needed if one is just interested in a combinatorial construction of open-closed TQFTs. For completeness, we nevertheless sketch how one can obtain the corresponding smoothing theorem: Let $K$ be a finite simplicial complex that triangulates an open-closed cobordism. Then every 1-simplex in the boundary is either black or coloured as we have explained above. The underlying polyhedron $|K|$ together with this partitioning of the boundary is already sufficient to read off the topological invariants defined in Section 3.2.4 of [12]. By the normal form of open-closed cobordisms of Definition 3.18 of [12], there exists an open-closed cobordism with the given invariants, and by Corollary 3.24 of [12], it is unique up to equivalence. 


\section{State Sum Construction}

We begin this section with an overview of the state sum construction in informal language.

Given a strongly separable symmetric Frobenius algebra $\left(A, \mu_{A}, \eta_{A}, \Delta_{A}, \varepsilon_{A}\right)$ in an Abelian symmetric monoidal category $\mathcal{C}$ and a connected open-closed cobordism $M$ with triangulation $T_{M}:|K| \rightarrow M$, we construct a morphism $Z(M)$ in $\mathcal{C}$.

For the duration of this section let $M$ be a connected open-closed cobordism with source $\partial_{0} M^{\text {in }}:=\vec{n}=\left(n_{1}, \cdots, n_{k}\right)$ and target $\partial_{0} M^{\text {out }}:=$ $\vec{n}^{\prime}=\left(n_{1}^{\prime}, \cdots, n_{k^{\prime}}^{\prime}\right)$. Let $j$ enumerate the black boundary components of $M$ so that $h_{j}$ denotes the number of 1 -simplices in the triangulation of the component $n_{j}$ for $1 \leq j \leq k$ or the component $n_{j}^{\prime}$ for $k+1 \leq j \leq k+k^{\prime}$. The number of 1 -simplices of $\partial_{0} M^{\text {in }}$ is given by the sum $m_{1}:=\sum_{j=1}^{k} h_{j}$, and the number of 1 -simplices of $\partial_{0} M^{\text {out }}$ by the sum $m_{2}:=\sum_{j=k+1}^{k+k^{\prime}} h_{j}$.

As a first step to constructing the morphism $Z(M)$, we construct a morphism $Z_{T_{M}}(M): A^{\otimes m_{1}} \rightarrow A^{\otimes m_{2}}$. These morphisms depend on the triangulation of the black boundary, but they are already invariant under bistellar moves and under elementary shellings of type (2.), i.e.

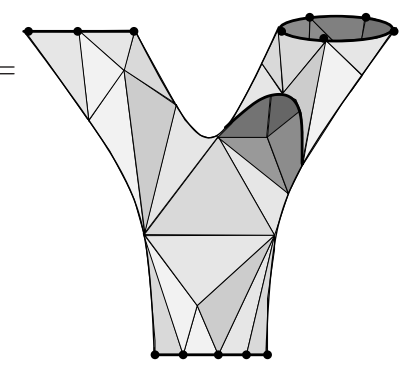

Figure 1: $\vec{n}=(1,0), \vec{n}^{\prime}=$ (1), $h_{1}=2, h_{2}=5, h_{3}=$ $4, m_{1}=7$, and $m_{2}=4$. those in which all the involved boundary edges are coloured.

Define the symbol $A^{\left(n_{j}\right)}$ corresponding to the boundary component $n_{j}$ to be $A$ if $n_{j}=1$ and $p(A)$ if $n_{j}=0$ and define $A^{\otimes \vec{n}}$ to be the ordered tensor product $\otimes_{j=1}^{k} A^{\left(n_{j}\right)}$. Likewise, we set $A^{\otimes \vec{n}^{\prime}}$ equal to the ordered tensor product $\bigotimes_{j=k+1}^{k+k^{\prime}} A^{\left(n_{j}^{\prime}\right)}$.

In Section 4.3, we show that the isomorphisms $P_{k k}\left(A^{\otimes k}\right) \cong A$ and $Q_{k k}\left(A^{\otimes k}\right) \cong p(A)$ of Corollary 2.24 correspond to triangulated cylinders over $I$ or $S^{1}$. We construct a map $Z(M): A^{\otimes \vec{n}} \rightarrow$ $A^{\otimes \overrightarrow{n^{\prime}}}$ using these isomorphisms and the morphism $Z_{T_{M}}(M)$. Since the claim of Corollary 2.24 is independent of $k$, and since the isomorphisms used in that corollary correspond to triangulated cylinders over $I$ or $S^{1}$, the invariance under bistellar moves and elementary shellings of type $(2$.) can be used to show independence of the boundary triangulation. The morphism $Z(M)$ is then also invariant under elementary shellings of type (1.), i.e. those involving the black boundary. $Z(M)$ is therefore independent of the triangulation and thus well-defined for the open-closed cobordism $M$.

One can verify explicitly that composition and disjoint union work as required, and so the state sum defines an open-closed TQFT $Z: \mathbf{2 C o b}^{\text {ext }} \rightarrow \mathcal{C}$. The objects of $\mathcal{C}$ associated with the interval and the circle are $A$ and $p(A)$, respectively, by construction. What is the knowledgeable Frobenius algebra that characterizes this TQFT?

In order to answer this question, we compute the morphisms of $\mathcal{C}$ associated with the generating open-closed cobordisms (1.3) and show that the open-closed TQFT is characterized by the knowledgeable Frobenius algebra of Theorem 2.22

\subsection{Defining the state sum}

We first describe how to construct the morphism $Z_{T_{M}}(M): A^{\otimes m_{1}} \rightarrow A^{\otimes m_{2}}$. It is defined by a string diagram in $\mathcal{C}$ obtained from the graph Poincaré dual to the triangulation, see Figure 2 By the coherence theorem for symmetric monoidal categories, it does not matter how one projects the Poincaré dual graph onto the drawing plane.

For every 2-simplex (triangle), we put a 'trilinear form' $g^{(3)}$ (c.f. (2.32)), and for every edge in the interior, we have an inverse bilinear form $g^{*}=\Delta_{A} \circ \eta_{A}$. Note that $g^{(3)}$ has a symmetry under the cyclic group $C_{3}$, but not in general under the symmetric group $S_{3}$, and so this assignment depends on the orientation.

For every edge on the coloured boundary $\partial_{1} M$, we put a unit $\eta_{A}$. For every interior 0 -simplex (vertex), we multiply the resulting morphism by the inverse $a^{-1}$ of the window element. Since $a^{-1}$ is central and the cobordism connected, it does not matter where in the diagram we do this. 


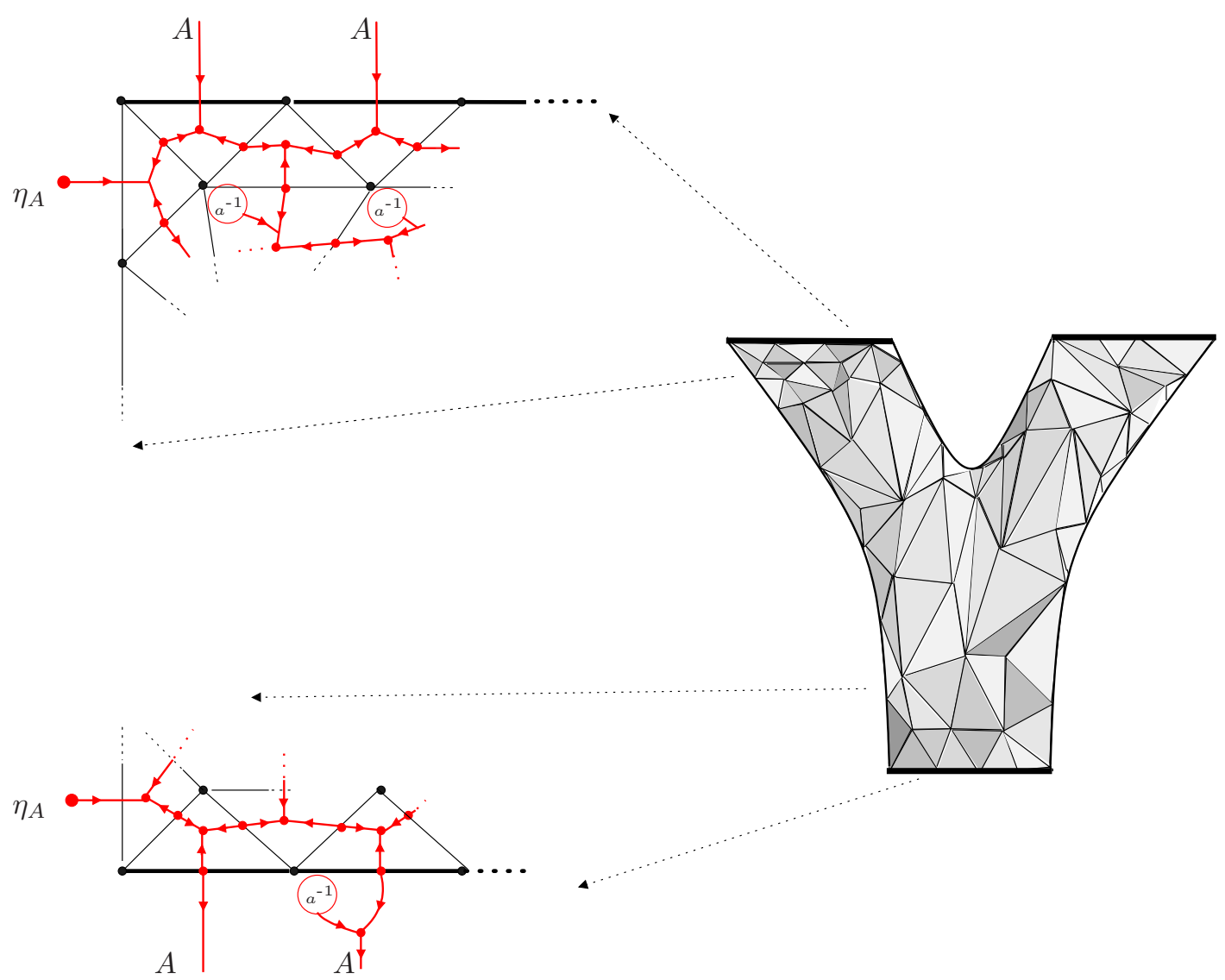

Figure 2: This figure illustrates the state sum for an open-closed cobordisms $M$.

At this stage, we have a morphism $A^{\otimes\left(m_{1}+m_{2}\right)} \rightarrow \mathbb{1}$ of $\mathcal{C}$. Finally, for every edge in the black out-boundary $\partial_{0} M^{\text {out }}$, we put a $g^{*}$, too, in order to turn this into a morphism $A^{\otimes m_{1}} \rightarrow A^{\otimes m_{2}}$. Then, for every vertex in the black out-boundary that is not a corner, we multiply by $a^{-1}$.

The terminology sum in 'state sum' is justified by the following point of view: If $\mathcal{C}=$ Vect $_{k}$ and if one chooses a basis of $A$ and expands all linear maps in this basis, the state sum contains a sum over the basis vectors for each edge in the interior of $M$. This is the sum involved in the state sum.

The morphisms specified by the string diagram have two important properties.

- Gluing triangulated open-closed cobordisms along a common black boundary that is triangulated with the same number of edges, corresponds to the composition of morphisms.

- The disjoint union of open-closed cobordisms gives the tensor product of morphisms.

The definition reads in detail as follows.

Definition 4.1. Let $\left(A, \mu_{A}, \eta_{A}, \Delta_{A}, \varepsilon_{A}\right)$ be a strongly separable symmetric Frobenius algebra in an Abelian symmetric monoidal category $\mathcal{C}$. Let $M$ be an open-closed cobordism with triangulation $T_{M}:|K| \rightarrow M$. Let $K^{(j)} \subseteq K$ denote the set of $j$-simplices, $j \in\{0,1,2\}$.

We characterize the edges, i.e. the elements $\sigma_{\{i, j\}} \in K^{(1)}$, by two-element sets $\{i, j\} \subseteq K^{(0)}$, $i \neq j$, of vertices. The oriented triangles $\sigma_{(i, j, k)} \in K^{(2)}$ are characterized by triples $(i, j, k) \in$ $K^{(0)} \times K^{(0)} \times K^{(0)}$ of vertices, modulo a permutation by a 3 -cycle.

We define the morphism $Z_{T_{M}}(M): A^{\otimes m_{1}} \rightarrow A^{\otimes m_{2}}$ as a composition

$$
Z_{T_{M}}(M):=Z_{T_{M}}^{(2)} \circ\left(a^{-k} \cdot \operatorname{id}_{A \otimes N}\right) \circ \tau \circ Z_{T_{M}}^{(1)} .
$$


where $N=m_{2}+\left|\left\{\sigma \in K^{(1)}: \sigma \subseteq \partial M\right\}\right|+2\left|\left\{\sigma \in K^{(1)}: \sigma \subseteq M \backslash \partial M\right\}\right|=m_{2}+3\left|K^{(2)}\right|$. The power of the inverse window element in (4.1) is $k=\left|\left\{\sigma \in K^{(0)}: \sigma \subseteq M \backslash \partial M\right\}\right|+\mid\left\{\sigma \in K^{(0)}: \sigma \subseteq\right.$ $\left.\partial_{0} M^{\text {out }} \backslash\left(\partial_{0} M \cap \partial_{1} M\right)\right\} \mid$ - the number of interior vertices plus the number of vertices on the outgoing edge that are not corners. We exploit the coherence theorem for monoidal categories and suppress the associativity and unit constraints of $\mathcal{C}$ and define

$$
Z_{T_{M}}^{(1)}:=\left(\bigotimes_{j=1}^{m_{1}} \mathrm{id}_{A}\right) \otimes\left(\bigotimes_{j=1}^{m_{2}} g^{*}\right) \otimes\left(\bigotimes_{\substack{\sigma \in K^{(1)} \\ \sigma \subseteq M \backslash \partial M}} g^{*}\right) \otimes\left(\bigotimes_{\substack{\sigma \in K^{(1)} \\ \sigma \subseteq \partial_{1} M}} \eta_{A}\right): A^{\otimes m_{1}} \rightarrow A^{\otimes N} .
$$

and

$$
Z_{T_{M}}^{(2)}:=\left(\bigotimes_{j=1}^{m_{2}} \operatorname{id}_{A}\right) \otimes\left(\bigotimes_{\sigma \in K^{(2)}} g^{(3)}\right): A^{\otimes N} \rightarrow A^{\otimes m_{2}},
$$

The morphism $\tau: A^{\otimes N} \rightarrow A^{\otimes N}$ permutes the tensor factors. In order to specify this permutation, we associate the factors of the target of 4.2) and those of the domain of (4.3) with the edges $\sigma_{\{i, j\}} \in K^{(1)}$. This is denoted by superscripts such as $A^{\{i, j\}}$. The permutation $\tau$ is specified by requiring that it maps each factor $A^{\{i, j\}}$ to one whose superscript is the same edge.

The superscripts for the A's in the target of (4.2) are as follows. We go through the factors of (4.2) from left to right.

- For every edge $\sigma_{\{i, j\}}$ in the black in-boundary $\partial_{0} M^{\text {in }}$, we have $\operatorname{id}_{A}: A \rightarrow A^{\{i, j\}}$. There are $m_{1}$ edges of this sort.

- For every edge $\sigma_{\{i, j\}}$ in the black out-boundary $\partial_{0} M^{\text {out }}$, we have $g^{*}: \mathbb{1} \rightarrow A^{\{i, j\}} \otimes A^{\{i, j\}}$. This edge therefore appears twice as a superscript, but due to the symmetry of $g^{*}$, we need not distinguish the two. There are $m_{2}$ edges of this sort.

- For every edge $\sigma_{\{i, j\}} \subseteq M \backslash \partial M$ in the interior, we have $g^{*}: \mathbb{1} \rightarrow A^{\{i, j\}} \otimes A^{\{i, j\}}$. Again the superscript occurs twice, and we do not distinguish.

- For every edge $\sigma_{\{i, j\}} \subseteq \partial_{1} M$ in the coloured boundary, we have $\eta_{A}: \mathbb{1} \rightarrow A^{\{i, j\}}$.

The superscripts for the $A$ 's in the domain of 4.3) are as follows.

- For every edge $\sigma_{\{i, j\}}$ in the black out-boundary $\partial_{0} M^{\text {out }}$, we have $\operatorname{id}_{A}: A^{\{i, j\}} \rightarrow A$.

- For every oriented triangle $\sigma_{(i, j, k)} \in K^{(2)}$, we have $g^{(3)}: A^{\{i, j\}} \otimes A^{\{j, k\}} \otimes A^{\{k, i\}} \rightarrow \mathbb{1}$. Due to the cyclic symmetry of the 'trilinear form' $g^{(3)}$, this morphism is invariant under permutations of the triple $(i, j, k)$ by a 3 -cycle.

Notice that the edges that appear as superscripts in the target of (4.2) and those in the domain of (4.3) agree including their multiplicities, and that the permutation $\tau$ is well defined.

See (4.7) for an example of the diagram produced by the state sum.

\subsection{Invariance under Pachner moves}

Proposition 4.2. For a connected open-closed cobordism $M$ with triangulation $T_{M}$, the state sum $Z_{T_{M}}(M)$ is invariant under the 1-3 and 2-2 Pachner moves and under the elementary shellings of type (2.).

Proof. The 2-2 Pachner move follows from the cyclic symmetry of the 'trilinear form' $g^{(3)}$.

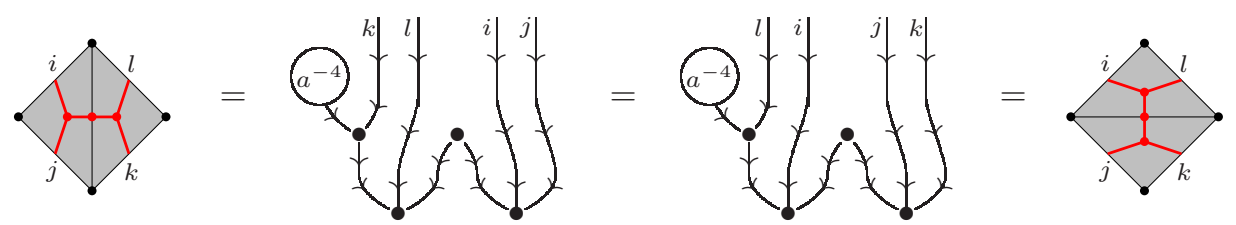


The 1-3 Pachner move is slightly more difficult because it involves subdividing a triangle which inserts an additional internal vertex. It makes use of the bubble move (2.48):

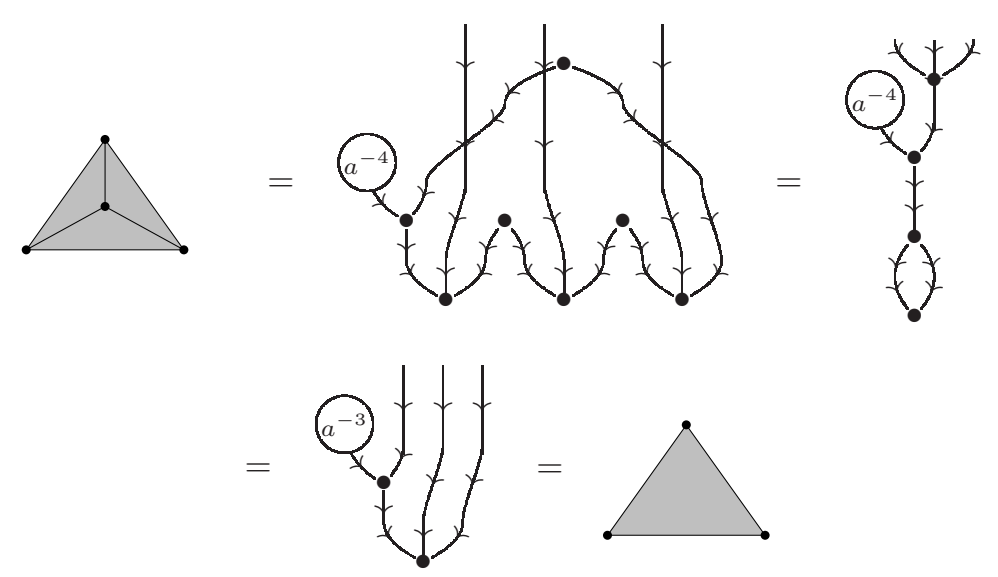

There are two elementary shellings (3.4) of type (2.). Recall that the state sum assigns to each edge of the coloured boundary the algebra unit $\eta_{A}: \mathbb{1} \rightarrow A$. The first move of (3.4) follows directly from the unit axioms. The second move turns an interior vertex

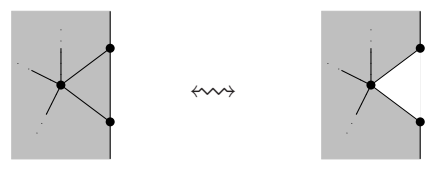
into an exterior vertex (featured to the right). This move follows from the bubble move (2.48):

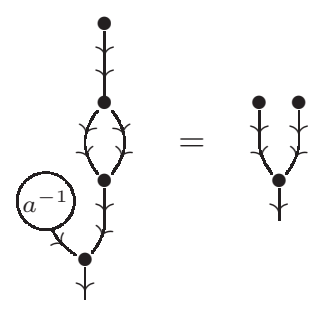

Note that the bubble move (2.48) is required to prove the above proposition. This is the reason why we cannot define the state sum for the non strongly separable algebras of Example 2.10 (2) and 2.11(2).

For convenience, we sometimes use degenerate triangulations in which the two vertices in the boundary of an edge agree. In this case it is always understood that we apply bistellar moves and elementary shellings in order to turn them into proper simplicial complexes.

An example showing the diagram produced by the state sum on the torus $T^{2}$ is depicted below:

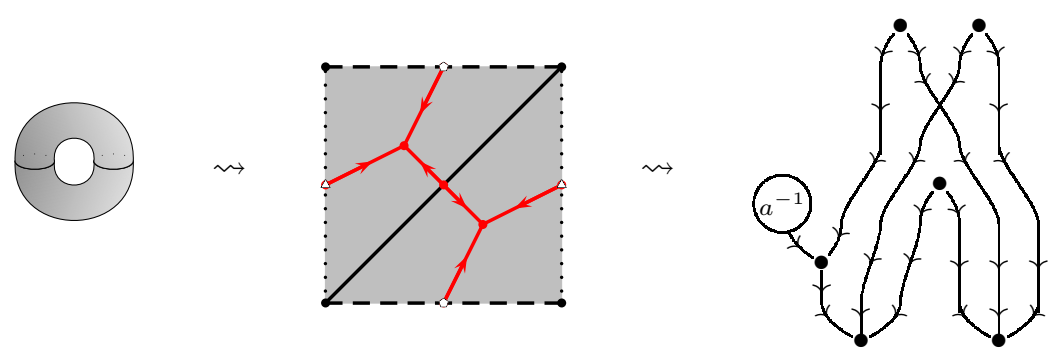

Here we have used the triangulation of the torus as a rectangle where the dotted and dashed lines are identified in the usual way. After the identifications this triangulation has a single interior vertex and hence the single factor of $a^{-1}$ that appears in the string diagram on the right. 


\subsection{Independence of the triangulation of black boundaries}

We now define a morphism $Z(M)$ from the morphism $Z_{T_{M}}(M)$ which does not depend on the choice of triangulation of the black boundary. Observe that to each black boundary component $n_{j}$ triangulated with $h_{j}$ edges, we have associated the vector space $A^{\otimes h_{j}}$.

Proposition 4.3. For the triangulations $T_{I \times I}^{k \ell}$ and $T_{S^{1} \times I}^{k \ell}$ of the flat strip $I \times I$ and the cylinder $S^{1} \times I$ with $\ell$ incoming edges and $k$ outgoing edges in their black boundaries, the state sum of Definition 4.1 yields the morphisms $P_{k \ell}: A^{\otimes \ell} \rightarrow A^{\otimes k}$ and $Q_{k \ell}: A^{\otimes \ell} \rightarrow A^{\otimes k}$ of Proposition 2.23 That is,

$$
\begin{aligned}
Z_{T_{I \times I}^{k \ell}}(I \times I) & =P_{k \ell}, \\
Z_{T_{S^{1} \times I}^{k \ell}}\left(S^{1} \times I\right) & =Q_{k \ell} .
\end{aligned}
$$

Proof. Write down the string diagram defining the state sum, c.f. Figure 2 and use the bubble move and the axioms of a symmetric Frobenius algebra.

We here include the simplest triangulations of $S^{1} \times I$ and $I \times I$ and the associated morphisms for $k=\ell=1$ :
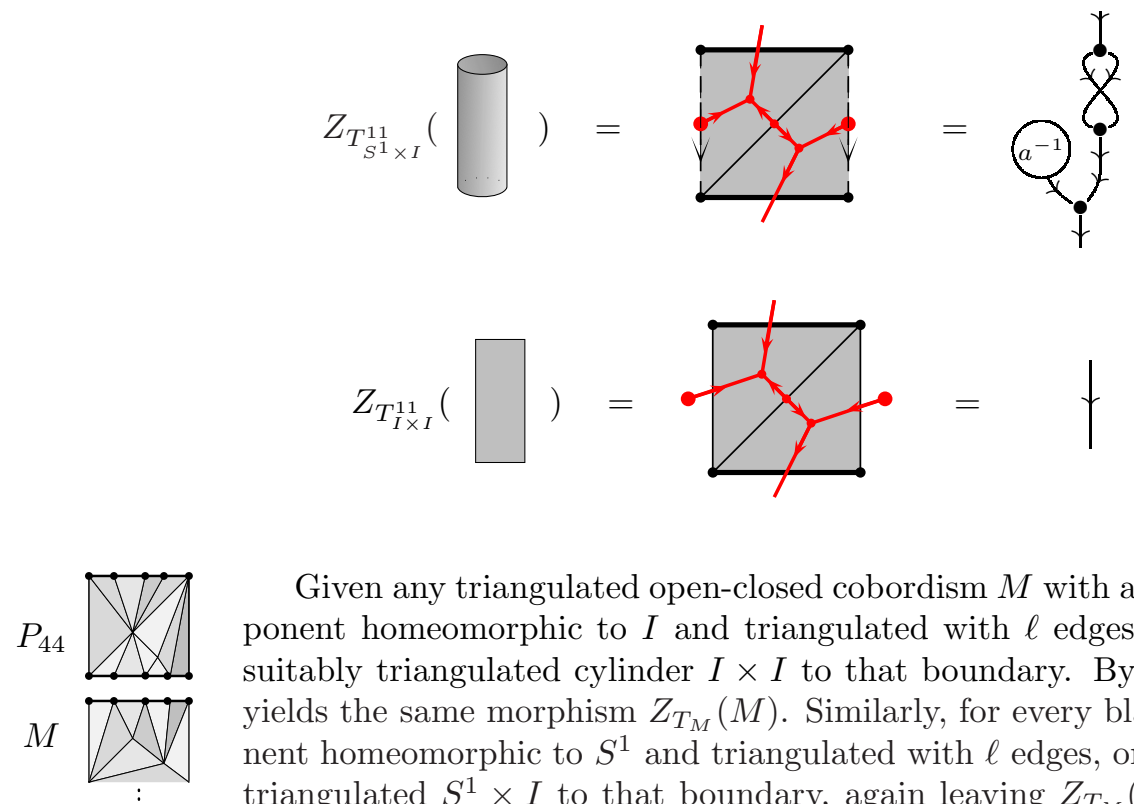

Given any triangulated open-closed cobordism $M$ with a black boundary component homeomorphic to $I$ and triangulated with $\ell$ edges, one can now glue a suitably triangulated cylinder $I \times I$ to that boundary. By Proposition 4.2 this yields the same morphism $Z_{T_{M}}(M)$. Similarly, for every black boundary component homeomorphic to $S^{1}$ and triangulated with $\ell$ edges, one can glue a suitably triangulated $S^{1} \times I$ to that boundary, again leaving $Z_{T_{M}}(M)$ unchanged. It is therefore sufficient to consider the restriction of $Z_{T_{M}}(M)$ to the appropriate images of the idempotents $P_{\ell \ell}$ and $Q_{\ell \ell}$, respectively. We therefore define:

Definition 4.4. For every open-closed cobordism $M$ with triangulation $T_{M}$, we define the state sum $\tilde{Z}_{T_{M}}(M)$ by subsequently pre- and post-composing $Z_{T_{M}}(M)$ with the following morphisms: For each $n_{j} \in \vec{n}=\partial_{0} M^{\text {in }}$ triangulated with $h_{j}$ edges, pre-composition with im $P_{h_{j} h_{j}}$ if $n_{j}=1$ and pre-composition with im $Q_{h_{j} h_{j}}$ if $n_{j}=0$; For each $n_{j}^{\prime} \in \vec{n}^{\prime}=\partial_{0} M^{\text {out }}$ triangulated with $h_{j}$ edges, post-composition with coim $P_{h_{j} h_{j}}$ if $n_{j}=1$ and post-composition with coim $Q_{h_{j} h_{j}}$ if $n_{j}=0$.

If we write $R_{k \ell}^{(0)}:=P_{k \ell}$ and $R_{k \ell}^{(1)}:=Q_{k \ell}$, then the above composite is the morphism

$$
\begin{aligned}
\tilde{Z}_{T_{M}}(M)= & \left(\bigotimes_{j=k+1}^{k+k^{\prime}} \operatorname{coim} R_{h_{j} h_{j}}^{\left(n_{j}\right)}\right) \circ Z_{T_{M}}(M) \circ\left(\bigotimes_{j=1}^{k} \operatorname{im} R_{h_{j} h_{j}}^{\left(n_{j}\right)}\right): \\
& \bigotimes_{j=1}^{k} R_{h_{j} h_{j}}^{\left(n_{j}\right)}\left(A^{\otimes h_{j}}\right) \rightarrow \bigotimes_{j=k+1}^{k+k^{\prime}} R_{h_{j} h_{j}}^{\left(n_{j}\right)}\left(A^{\otimes h_{j}}\right) .
\end{aligned}
$$


One can now use the isomorphisms of Corollary2.24 in order to relate the $\tilde{Z}_{T_{M}}(M)$ for different triangulations of the black boundary as follows. The morphism $\tilde{Z}_{T_{M}}(M)$ is completely determined by the triangulation of the boundary $\partial_{0} M$ by Proposition 4.2 Hence, the morphism $\tilde{Z}_{T_{M}}(M)$ associated to a triangulation $T_{M}$ is related to the morphism $\tilde{Z}_{T_{M}^{\prime}}(M)$ obtained from a different triangulation $T_{M}^{\prime}$ by gluing on cylinders whose boundaries are appropriately triangulated. These cylinders yield precisely the morphisms $P_{k \ell}$ and $Q_{k \ell}$.

Definition 4.5. For every open-closed cobordism $M$, we choose a triangulation $T_{M}$. We define the state sum $Z(M)$ by subsequently pre- and post-composing $\tilde{Z}_{T_{M}}(M)$ with the following morphisms: For each $n_{j} \in \vec{n}=\partial_{0} M^{\text {in }}$ triangulated with $h_{j}$ edges, pre-composition with $\Phi_{h_{j}}$ if $n_{j}=1$ and pre-composition with $\Psi_{h_{j}}$ if $n_{j}=0$; For each $n_{j}^{\prime} \in \vec{n}^{\prime}=\partial_{0} M^{\text {out }}$ triangulated with $h_{j}$ edges, post-composition with $\Phi_{h_{j}}^{-1}$ if $n_{j}=1$ and post-composition with $\Psi_{h_{j}}^{-1}$ if $n_{j}=0$. This yields the morphism

$$
Z(M)=\left(\bigotimes_{j=k+1}^{k+k^{\prime}}\left(\Xi_{h_{j}}^{\left(n_{j}\right)}\right)^{-1}\right) \circ \tilde{Z}_{T_{M}}(M) \circ\left(\bigotimes_{j=1}^{k} \Xi_{h_{j}}^{\left(n_{j}\right)}\right): A^{\otimes \vec{n}} \rightarrow A^{\otimes \vec{n}^{\prime}},
$$

where we write $\Xi_{h_{j}}^{(0)}:=\Psi_{h_{j}}$ and $\Xi_{h_{j}}^{(1)}:=\Phi_{h_{j}}$.

The definition of $Z(M)$ is illustrated below:
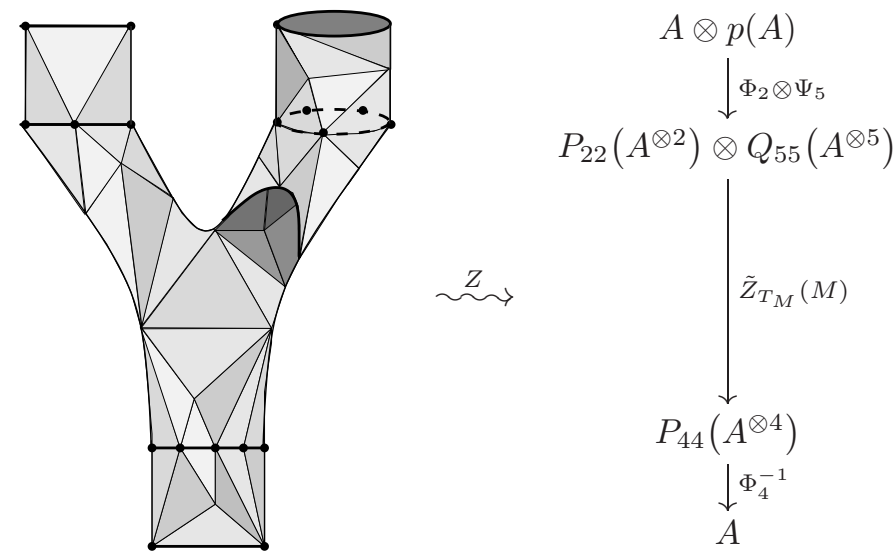

Theorem 4.6. The morphism (4.13) is well defined, i.e. it does not depend on the triangulation $T_{M}$ of $M$. In particular, it is independent of the numbers $h_{j}$ of edges in Definition 4.4 and Definition 4.5.

Proof. Insert (4.12) into (4.13) and draw the cylinders over $I$ and over $S^{1}$ whose triangulations are given by $\operatorname{im} P_{h_{j} h_{j}} \circ \Phi_{h_{j}}=P_{h_{j} 1}$, etc. and glue them to the triangulation used in the state sum $Z_{T_{M}}(M)$ of Definition 4.1 The invariance under bistellar moves and elementary shellings of type (2.) of Proposition 4.2 then implies the theorem.

\subsection{Open-closed Topological Quantum Field Theories}

From Definition 4.1 it is obvious that the state sum $Z(M)$ associates with the composition of open-closed cobordisms the composition of morphisms of $\mathcal{C}$ and with the disjoint union of openclosed cobordisms the tensor product of morphisms in $\mathcal{C}$. It is not difficult to see that we get a symmetric monoidal functor $Z: \mathbf{2} \mathbf{C o b}^{\text {ext }} \rightarrow \mathcal{C}$, i.e. an open-closed TQFT.

In this section, we show that this open-closed TQFT is the one characterized by the knowledgeable Frobenius algebra of Theorem 2.22 


\subsubsection{Generators via the state sum construction}

Below we provide a choice of triangulation for some of the generators in $\mathbf{2} \mathbf{C o b}^{\mathrm{ext}}$.
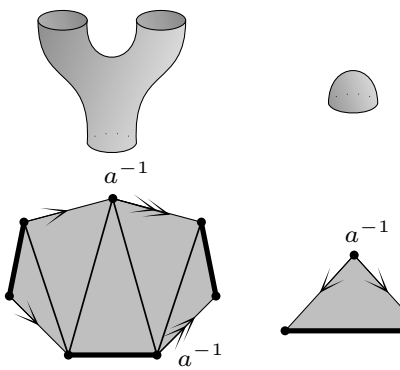
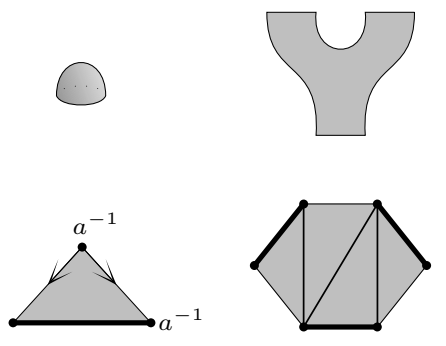
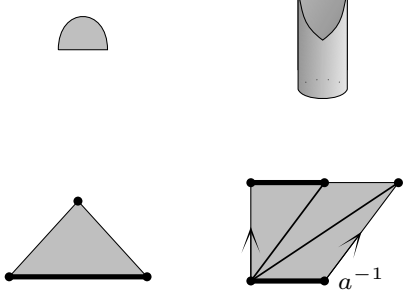

Those edges with matching arrow heads on the triangulations are to be identified. The black boundaries are depicted slightly thicker than the coloured boundaries. A choice of triangulation for the remaining generators is immediate from those above. The factors of $a^{-1}$ are meant to remind the reader which vertices in the triangulation contribute factors of $a^{-1}$.

Using these triangulations we can compute the morphisms $Z_{T_{M}}(M)$ associated to the openclosed cobordisms $M$ generating $\mathbf{2} \mathbf{C o b}^{\text {ext }}$. For completeness, we include the triangulation of the cylinders $S^{1} \times I$ and $I \times I$ as well.

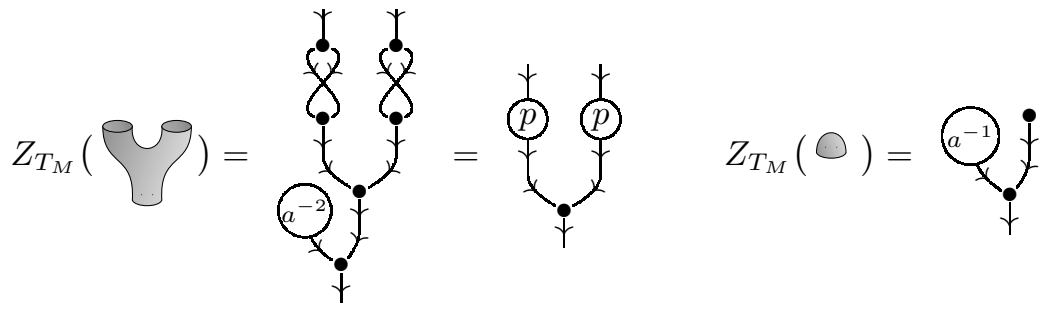

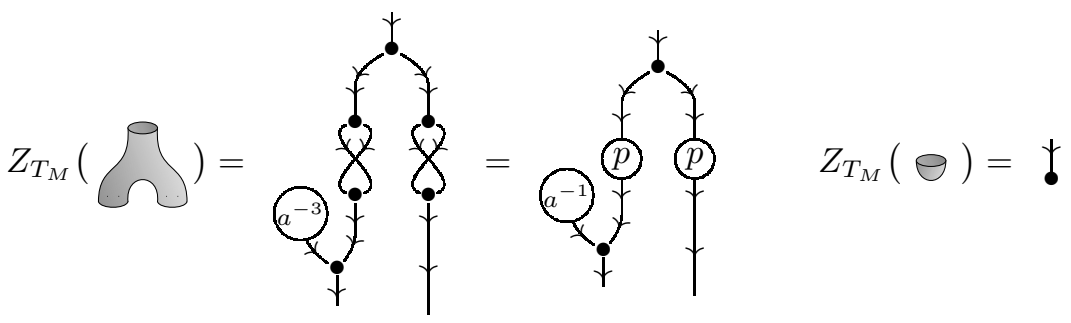

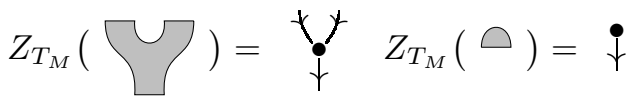

$$
\begin{aligned}
& Z_{T_{M}}(\Omega)=\underset{f}{\dagger} Z_{T_{M}}(\nabla)=\text { ‘ }
\end{aligned}
$$

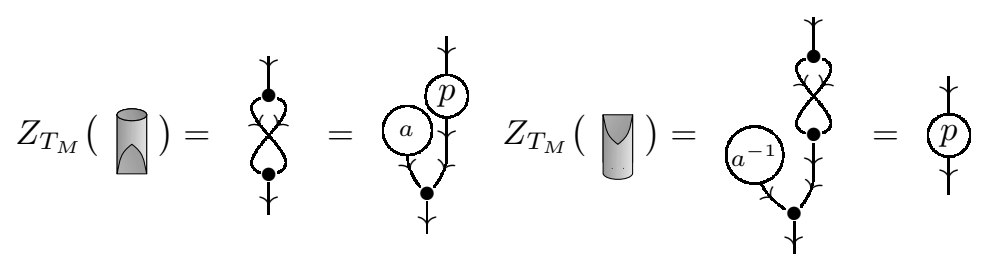

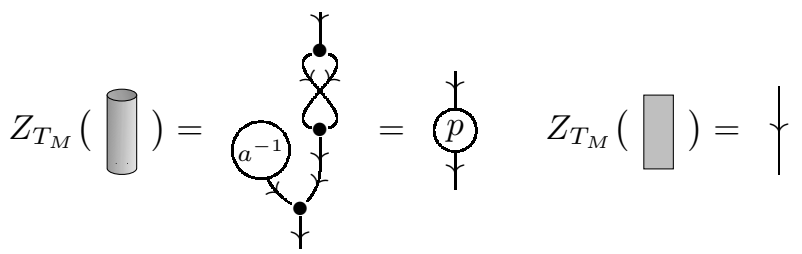


Theorem 4.7. Let $\mathcal{C}$ be an Abelian symmetric monoidal category and $A$ be a rigid and strongly separable algebra object in $\mathcal{C}$ that is equipped with the structure of a symmetric Frobenius algebra. Then the state sum (4.1) defines an open-closed TQFT $Z: 2 \mathbf{C o b}^{\text {ext }} \rightarrow \mathcal{C}$. It is characterized by the knowledgeable Frobenius algebra constructed from $A$ in Theorem 2.22

Proof. Using the triangulations of the generators given in (4.16)-4.21), compute the morphisms $Z_{T_{M}}(M)$ for each generator of $\mathbf{2} \mathbf{C o b}^{\mathrm{ext}}$. Pre and post composing with the relevant maps specified in Definitions 4.4 and 4.5 produces the knowledgeable Frobenius algebra $\left(A, Z(A), \imath, \imath^{*}\right)$ defined in Theorem 2.22. For example, $Z_{T_{M}}(\zeta)=\mu_{A} \circ(p \otimes p)$ so that $\tilde{Z}_{T_{M}}(\rho)=\operatorname{coim} Q_{11} \circ \mu_{A} \circ$ $(p \otimes p) \circ\left(\operatorname{im} Q_{11} \otimes \operatorname{im} Q_{11}\right)$. Noting that $Q_{11}=p$ and using the image factorization of $p$ (2.54) together with the idempotent property $p^{2}=p$ it is easy to check that

$$
Z(\varrho)=\operatorname{coim} p \circ \mu_{A} \circ(\operatorname{im} p \otimes \operatorname{im} p)
$$

as specified in Theorem 2.22 ,

Since $\mathbf{2} \mathbf{C o b}^{\text {ext }}$ is the strict symmetric monoidal category freely generated by a knowledgeable Frobenius algebra object, this uniquely determines a symmetric monoidal functor $Z: 2 \mathbf{C o b}^{\text {ext }} \rightarrow$ $\mathcal{C}$.

Recall that given an open-closed TQFT, the algebra object $A:=Z(I)$ does not necessarily determine the object $C:=Z\left(S^{1}\right)$. Consider, for example, the knowledgeable Frobenius algebra $\left(A, C, \imath, \imath^{*}\right)$ of Example 2.19] in which $C \not Z Z(A)$, and secondly the knowledgeable Frobenius algebra $\left(A, Z(A), \imath^{\prime}, \imath^{\prime *}\right)$ constructed in Theorem 2.22 based on the same $A$. Both characterize an open-closed TQFT, but only the latter one can be obtained from the state sum.

Conversely, in an open-closed TQFT, the object $Z\left(S^{1}\right)$ does not determine the object $Z(I)$. This can be easily seen from Example 4.8 below.

\subsection{Examples}

In [12] it was shown that connected open-closed cobordisms are determined up to orientationpreserving diffeomorphism preserving the black boundary by a set of topological invariants defined in the work of Baas, Cohen, and Ramírez [34]. These topological invariants are the genus (defined as the genus of the underlying topological 2-manifold), the window number, defined as the number of components of $\partial_{1} M$ diffeomorphic to $S^{1}$, and the boundary permutation. For a surface $M$ $\left(\partial_{0} M=\emptyset\right)$ only the genus and window number are relevant. In this context we will refer to the window number as the number of punctures in $M$.

Let $\left(A, C, \imath, \imath^{*}\right)$ be a knowledgeable Frobenius algebra in a symmetric monoidal category $\mathcal{C}$. We call $\mu_{C} \circ \Delta_{C}: C \rightarrow C$ the genus-one operator and $\imath^{*} \circ \imath: C \rightarrow C$ the window operator. The invariant associated to the connected surface $M_{k}^{\ell}$ of genus $\ell$ with $k$ punctures is determined by evaluating the morphism

$$
Z\left(M_{k}^{\ell}\right)=\varepsilon_{C} \circ\left(\imath^{*} \circ \imath\right)^{k} \circ\left(\mu_{C} \circ \Delta_{C}\right)^{\ell} \circ \eta_{C}: \mathbb{1} \rightarrow \mathbb{1}
$$

in $\mathcal{C}$.

In this section, we provide several examples of strongly separable symmetric Frobenius algebras and use the genus-one operator and the window operator to compute the state sum invariant $Z\left(M_{k}^{\ell}\right)$.

Example 4.8. Let $k$ be a field, $n \in \mathbb{N}$, and $m_{1}, \ldots, m_{n} \in \mathbb{N}$, and consider the direct product ${ }^{7}$

$$
A:=\bigoplus_{j=1}^{n} M_{m_{j}}(k)
$$

of matrix algebras. We choose a basis $\left\{e_{p q}^{(j)}\right\}_{1 \leq p, q \leq m_{j}, 1 \leq j \leq n}$ of $A$ such that the multiplication reads $\mu_{A}\left(e_{p q}^{(j)} \otimes e_{r s}^{(\ell)}\right)=\delta_{j \ell} \delta_{r q} e_{p s}^{(j)}$ with unit $\eta_{A}(1)=\sum_{j=1}^{n} \sum_{p=1}^{m_{j}} e_{p p}^{(j)}$. The $k$-algebra $\left(A, \mu_{A}, \eta_{A}\right)$

\footnotetext{
${ }^{7}$ We write $\oplus$ because this is actually the biproduct in the Abelian category Vect $_{k}$.
} 
is strongly separable if and only if for all $j$, char $k$ does not divide $m_{j}$. From now on we assume that this condition holds.

The centre $Z(A)$ of $A$ has a basis $\left\{z_{j}\right\}_{1 \leq j \leq n}$ of orthogonal idempotents $z_{j}:=\sum_{p=1}^{m_{j}} e_{p p}^{(j)}$, i.e. $\mu_{A}\left(z_{j} \otimes z_{\ell}\right)=\delta_{j \ell} z_{j}$. The symmetric Frobenius algebra structures $\left(A, \mu_{A}, \eta_{A}, \Delta_{A}, \varepsilon_{A}\right)$ are characterized by the invertible central elements $a=\sum_{j=1}^{n} a_{j} z_{j}$, i.e. $a_{j} \in k \backslash\{0\}$ for all $j$, as follows:

$$
\begin{aligned}
\Delta_{A}\left(e_{p q}^{(j)}\right) & =a_{j} m_{j}^{-1} \sum_{r=1}^{m_{j}} e_{p r}^{(j)} \otimes e_{r q}^{(j)} \\
\varepsilon_{A}\left(e_{p q}^{(j)}\right) & =\delta_{p q} m_{j} a_{j}^{-1}
\end{aligned}
$$

and indeed one finds $\left(\mu_{A} \circ \Delta_{A} \circ \eta_{A}\right)(1)=a$ for the window element. This illustrates further the distinction between special Frobenius algebras and strongly separable Frobenius algebras. $A$ is special if and only if $a_{i}=a_{j}$ for all $i, j$. We compute the idempotent $p$ of (2.53) as follows:

$$
p\left(e_{p q}^{(j)}\right)=\delta_{p q} m_{j}^{-1} \sum_{r=1}^{m_{j}} e_{r r}^{(j)}
$$

and indeed the image is $p(A) \cong Z(A)$ with the splitting

$$
\begin{aligned}
\operatorname{im} p: p(A) \rightarrow A, \quad z_{j} & \mapsto \sum_{p=1}^{m_{j}} e_{p p}^{(j)}, \\
\operatorname{coim} p: A \rightarrow p(A), \quad e_{p q}^{(j)} & \mapsto \delta_{p q} m_{j}^{-1} z_{j}
\end{aligned}
$$

The knowledgeable Frobenius algebra $\left(A, C, \imath, \imath^{*}\right)$ of Theorem 2.22 for this algebra $A$ is given by the following commutative Frobenius algebra structure $\left(C, \mu_{C}, \eta_{C}, \Delta_{C}, \varepsilon_{C}\right)$ on $C:=Z(A)$ :

$$
\begin{aligned}
\mu_{C}\left(z_{j} \otimes z_{\ell}\right) & =\delta_{j \ell} z_{j}, \\
\eta_{C}(1) & =\sum_{j=1}^{n} z_{j}, \\
\Delta_{C}\left(z_{j}\right) & =a_{j}^{2} m_{j}^{-2} z_{j} \otimes z_{j}, \\
\varepsilon_{C}\left(z_{j}\right) & =m_{j}^{2} a_{j}^{-2},
\end{aligned}
$$

together with

$$
\begin{aligned}
\imath: C \rightarrow A, \quad z_{j} & \mapsto \sum_{p=1}^{m_{j}} e_{p p}^{(j)}, \\
\imath^{*}: A \rightarrow C, \quad e_{p q}^{(j)} & \mapsto a_{j} m_{j}^{-1} \delta_{p q} z_{j} .
\end{aligned}
$$

We finally compute the genus-one operator $\left(\mu_{C} \circ \Delta_{C}\right)\left(z_{j}\right)=a_{j}^{2} m_{j}^{-2} z_{j}$ and the window operator $\left(\imath^{*} \circ \imath\right)\left(z_{j}\right)=a_{j} z_{j}$, and so the invariant (4.36) associated with the genus $\ell$-surface with $k$ punctures, $k, \ell \in \mathbb{N}_{0}$, is

$$
Z\left(M_{k}^{\ell}\right)(1)=\left(\varepsilon_{C} \circ\left(\imath^{*} \circ \imath\right)^{k} \circ\left(\mu_{C} \circ \Delta_{C}\right)^{\ell} \circ \eta_{C}\right)(1)=\sum_{j=1}^{n} a_{j}^{k+2(\ell-1)} m_{j}^{-2(\ell-1)}
$$

Fukuma-Hosono-Kawai [6] choose the canonical Frobenius algebra structure on A, i.e. $a=\eta$ and therefore $a_{j}=1$ for all $j$. In this case, the invariant is blind to the window number $k$. With a generic symmetric Frobenius algebra structure, however, one can easily obtain an invariant that can distinguish any two inequivalent connected surfaces. 
Example 4.9. Let $G$ be a finite group, $k$ a field, and $A:=k[G]$ be the group algebra. We choose the basis $\{g\}_{g \in G}$ for $A$ and have $\mu_{A}(g \otimes h)=g h$ for $g, h \in G$ and $\eta_{A}(1)=e$. The $k$-algebra $\left(A, \mu_{A}, \eta_{A}\right)$ is strongly separable if and only if char $k$ does not divide the order $|G|$ of $G$. We now assume that this condition holds.

We denote by $[g]:=\left\{h g h^{-1}: h \in G\right\} \subseteq G$ the conjugacy class of $g \in G$ and by $G / \sim:=$ $\{[g]: g \in G\}$ the set of classes. Then the centre $Z(A)$ has the basis $\left\{z_{[g]}\right\}_{[g] \in G / \sim}$ where $z_{[g]}:=$ $\sum_{h \in[g]} h$ denotes the class sum. We have the unit $\eta_{A}(1)=\sum_{[g] \in G / \sim} z_{[g]}$ and $\mu_{A}\left(z_{[g]} \otimes z_{[h]}\right)=$ $\sum_{[\ell] \in G / \sim} \mu_{[g],[h]}^{[\ell]} z_{[\ell]}$ for all $g, h \in G$ with some $\mu_{[g],[h]}^{[\ell]} \in k$.

The $z_{[g]}$ are in general not orthogonal idempotents. Working with a generic invertible central element in the basis $\left\{z_{[g]}\right\}_{[g] \in G / \sim}$ is not very instructive. If $k$ is algebraically closed, the irreducible characters $\chi_{\rho}: G \rightarrow k$ provide us with a basis $\left\{z_{\rho}\right\}_{\rho}$ of orthogonal idempotents $z_{\rho}:=$ $d_{\rho}|G|^{-1} \sum_{g \in G} \chi_{\rho}(g) g, d_{\rho}=\chi_{\rho}(e)$, for $Z(A)$. We then get the same results as for a direct product of $d_{\rho} \times d_{\rho}$-matrix algebras.

In the following, we restrict ourselves to the symmetric Frobenius algebra structure

$$
\begin{aligned}
\Delta_{A}(g) & =\sum_{h \in G} h \otimes h^{-1} g \\
\varepsilon_{A}(g) & = \begin{cases}1, & \text { if } g=e \\
0, & \text { else }\end{cases}
\end{aligned}
$$

which is characterized by the window element $\left(\mu_{A} \circ \Delta_{A} \circ \eta_{A}\right)(1)=|G| e=|G| \eta_{A}(1)$. The symmetric Frobenius algebra $\left(A, \mu_{A}, \eta_{A}, \Delta_{A}, \varepsilon_{A}\right)$ is therefore special in the sense of (2.21). In this case

$$
\begin{aligned}
g^{*}(1)=\left(\Delta_{A} \circ \mu_{A}\right)(1) & =\sum_{h \in G} h \otimes h^{-1}, \\
g^{(3)}((g \otimes h) \otimes \ell)=\left(\varepsilon_{A} \circ \mu_{A} \circ\left(\mu_{A} \otimes \mathrm{id}_{A}\right)\right)((g \otimes h) \otimes \ell) & = \begin{cases}1, & \text { if } g h \ell=e \\
0, & \text { else }\end{cases}
\end{aligned}
$$

The state sum $Z(M)$ then agrees with the partition function of a topological gauge theory with gauge group $G$ or, in other words, with the volume of the moduli space of flat $G$-bundles on $M$. In the state sum of Definition [4.1 the window element $|G|$ is divided out for every vertex in the interior of $M$ (this prefactor of $Z(M)$ is sometimes called the anomaly). In the closed TQFT, the meaning of this factor is somewhat mysterious - the factor is merely needed in order to make the 1-3 Pachner move work - but in our extension to the open-closed TQFT, the factor $|G|$ is directly related to the symmetric Frobenius algebra structure of $A$ and thereby to topology.

Remark 4.10. Although our state sum of Definition 4.1 requires an oriented 2-manifold, the previous example with the group algebra $A=k[G]$ makes sense even for unoriented manifolds (without boundary). This is possible because $A$ also has the structure of an involutory Hopf algebra $\left(A, \mu_{A}, \eta_{A}, \Delta_{A}^{\text {Hopf }}, \varepsilon_{A}^{\text {Hopf }}, S_{A}\right)$ with

$$
\begin{aligned}
\Delta_{A}^{\operatorname{Hopf}}(g) & =g \otimes g, \\
\varepsilon_{A}^{\mathrm{Hopf}}(g) & =1, \\
S_{A}(g) & =g^{-1},
\end{aligned}
$$

with a co-integral $\sum_{g \in G} g$ and an integral $g \mapsto \delta_{G}(g)$ where $\delta_{G}(e)=1$ and $\delta_{G}(g)=0$ for all $g \neq e$. For this involutory Hopf algebra, one can evaluate Kuperberg's 3-manifold invariant [24] which does not refer to the 3 -simplices and therefore makes sense for (unoriented) 2-manifolds, too. In the oriented case, it agrees with our state sum. The unoriented case is treated in more generality in [11]. 


\section{$5 \quad$ State sums with D-branes}

Our next example, the groupoid algebra of a finite groupoid, also yields the state sum of an open-closed TQFT in a straightforward way, but in addition it provides us with an example of an $S$-coloured open-closed TQFT, c.f. Section 5 of [12].

A groupoid $\mathcal{G}=\left(X, G, s, t, \imath, \circ,{ }^{-1}\right)$ consists of sets $X$ (objects) and $G$ (morphisms) and maps $s: G \rightarrow X$ (source), $t: G \rightarrow X$ (target), $\imath: X \rightarrow G$ (identity), $\circ: G_{t} \times_{s} G:=\left\{\left(h_{1}, h_{2}\right) \in G \times\right.$ $\left.G: t\left(h_{1}\right)=s\left(h_{2}\right)\right\} \rightarrow G$ (composition, written from left to right) and ${ }^{-1}: G \rightarrow G$ (inversion) such that the following conditions are satisfied,

1. $s(\imath(x))=x$ and $t(\imath(x))=x$ for all $x \in X$,

2. $s\left(h_{1} \circ h_{2}\right)=s\left(h_{1}\right)$ and $t\left(h_{1} \circ h_{2}\right)=t\left(h_{2}\right)$ for all $\left(h_{1}, h_{2}\right) \in X_{t} \times_{s} X$,

3. $\left(h_{1} \circ h_{2}\right) \circ h_{3}=h_{1} \circ\left(h_{2} \circ h_{3}\right)$ for all $h_{1}, h_{2}, h_{3} \in G$ for which $t\left(h_{1}\right)=s\left(h_{2}\right)$ and $t\left(h_{2}\right)=s\left(h_{3}\right)$,

4. $\imath(s(h)) \circ h=h=h \circ \imath(t(h))$ for all $h \in G$,

5. $s\left(h^{-1}\right)=t(h)$ and $t\left(h^{-1}\right)=s(h)$ for all $h \in G$,

6. $h^{-1} \circ h=\imath(t(h))$ and $h \circ h^{-1}=\imath(s(h))$ for all $h \in G$.

The groupoid is called finite if $G$ is a finite set. For every $x \in X$, we denote its connected component by $[x]:=\{t(h): h \in G, s(h)=x\}$. The groupoid is called connected if $X=[x]$ for some $x \in X$. For $x \in X$, the star of $\mathcal{G}$ at $x$ is the set,

$$
\operatorname{st}_{\mathcal{G}}(x)=\{g \in G: s(g)=x\} .
$$

We denote the order of the star of $\mathcal{G}$ at $x \in X$ by $N_{[x]}:=\left|\operatorname{st}_{\mathcal{G}}(x)\right|$. It depends only on the connected component $[x]$ of $x \in X$.

Given a finite groupoid $\mathcal{G}=\left(X, G, s, t, \imath, \circ,{ }^{-1}\right)$ and a field $k$, the groupoid algebra $(k[G], \mu, \eta)$ is the free vector space $k[G]$ on the set of morphisms with the operations,

$$
\begin{aligned}
\mu\left(h_{1} \otimes h_{2}\right) & = \begin{cases}h_{1} \circ h_{2}, & \text { if } t\left(h_{1}\right)=s\left(h_{2}\right) \\
0, & \text { else }\end{cases} \\
\eta(1) & =\sum_{x \in X} \imath(x),
\end{aligned}
$$

where $h_{1}, h_{2} \in G$.

Example 5.1. Let $(G)=\left(X, G, s, t, \imath, \circ,{ }^{-1}\right)$ be a finite groupoid and consider the groupoid algebra $A:=k[G]$. The $k$-algebra $A$ is strongly separable if and only of char $k$ does not divide $N_{[x]}$ for any $x \in X$. From now on, we assume that this is the case.

We denote by $G^{(0)}:=\{g \in G: s(g)=t(g)\} \subseteq G$ the set of automorphisms, by $[g]:=$ $\left\{h \circ g \circ h^{-1}: h \in G, t(h)=t(g)\right\}$ the conjugacy class of the automorphism $g \in G^{(0)}$, and by $G^{(0)} / \sim:=\left\{[g]: g \in G^{(0)}\right\}$ the set of conjugacy classes. Choose the basis $\{h\}_{h \in G}$ of $A$. We find the centre $Z(A) \cong k\left[G^{(0)} / \sim\right]$ with a basis $\left\{z_{[g]}\right\}_{g \in G^{(0)} / \sim}$ where $z_{[g]}:=\sum_{h \in[g]} h$ denotes the class sum.

The canonical symmetric Frobenius algebra structure $\left(A, \mu_{A}, \eta_{A}, \Delta_{A}, \varepsilon_{A}\right)$ is given by

$$
\begin{aligned}
\varepsilon_{A}(g) & = \begin{cases}N_{[s(g)]}, & \text { if } g=\imath(s(g)) \\
0, & \text { else }\end{cases} \\
\Delta_{A}(g) & =\frac{1}{N_{[t(g)]}} \sum_{h \in G: s(h)=s(g)} h \otimes\left(h^{-1} \circ g\right),
\end{aligned}
$$


from which we obtain the canonical idempotent (2.53)

$$
p(g)= \begin{cases}z_{[g]} / N_{[t(g)]}, & \text { if } t(g)=s(g) \\ 0, & \text { else }\end{cases}
$$

with the image decomposition

$$
\begin{aligned}
& \operatorname{im} p: Z(A) \rightarrow A, \quad z_{[g]} \mapsto \sum_{h \in[g]} h, \\
& \operatorname{coim} p: A \rightarrow Z(A), \quad g \mapsto \begin{cases}z_{[g]} / N_{[t(g)]}, & \text { if } s(g)=t(g) \\
0, & \text { else. }\end{cases}
\end{aligned}
$$

From these data, one can compute the knowledgeable Frobenius algebra $\left(A, Z(A), \imath, \imath^{*}\right)$ that appears in Theorem 2.22 with $\imath=\operatorname{im} p$ and $\imath^{*}=\operatorname{coim} p$. The state sum construction therefore yields the corresponding open-closed TQFT.

There is, however, another point of view according to which the groupoid algebra gives rise to an $X$-coloured knowledgeable Frobenius algebra (Section 5 of [12]). Although this example is rather trivial, it nicely illustrates where the various structures appear.

Example 5.2. Let $\mathcal{G}=\left(X, G, s, t, \imath, \circ,{ }^{-1}\right)$ be a finite groupoid and $k$ be a field such that char $k$ does not divide $N_{[x]}$ for any $x \in X$. Denote by $\operatorname{Hom}(x, y)=\{g \in G: s(g)=x, t(g)=y\}$ the morphisms from $x$ to $y$. Then there is a family of vector spaces $A_{x y}:=k[\operatorname{Hom}(x, y)]$. By restricting the operations of the groupoid algebra $A=k[G]$ to the $A_{x y}$, we obtain the following linear maps:

$$
\begin{aligned}
& \mu_{x y z}: A_{x y} \otimes A_{y z} \rightarrow A_{x z}, \quad g_{1} \otimes g_{2} \mapsto g_{1} \circ g_{2}, \\
& \eta_{x}(1): k \rightarrow A_{x x}, \quad 1 \quad \mapsto \imath(x), \\
& \Delta_{x y z}: A_{x z} \rightarrow A_{x y} \otimes A_{y z}, \quad g \quad \mapsto \frac{1}{N_{[t(g)]}} \sum_{h \in G: s(h)=x} h \otimes h^{-1} \circ g, \\
& \varepsilon_{x}: A_{x x} \rightarrow k, \quad g \quad \mapsto \begin{cases}N_{[s(g)]}, & \text { if } g=\imath(x) \\
0, & \text { else }\end{cases}
\end{aligned}
$$

for $x, y, z \in X$. Similarly by restricting $\imath$ and $\imath^{*}$, we find for all $x \in X$ :

$$
\begin{aligned}
& \imath_{x}: Z(A) \rightarrow A_{x x}, z_{[g]} \mapsto \sum_{h \in[g]:} \sum_{h \in \operatorname{Hom}(x, x)} h, \\
& \imath^{*}: A_{x x} \rightarrow Z(A), g \mapsto \quad \frac{1}{N_{[x]}} z_{[g]} .
\end{aligned}
$$

Then we have an $X$-coloured knowledgeable Frobenius algebra

$$
\left(\left\{A_{x y}\right\},\left\{\mu_{x y z}\right\},\left\{\eta_{x}\right\},\left\{\Delta_{x y z}\right\},\left\{\varepsilon_{x}\right\}, Z(A),\left\{\imath_{x}\right\},\left\{\imath_{x}^{*}\right\}\right) .
$$

The commutative Frobenius algebra structure of $Z(A)$ is as in Theorem 2.22 In particular, each $A_{x x}, x \in X$, forms a symmetric Frobenius algebra, the $\imath_{x}: Z(A) \rightarrow A_{x x}$ are algebra homomorphisms, and each $A_{x y}$ forms an $\left(A_{x x}, A_{y y}\right)$-bimodule with dual $A_{y x}$. Observe that the state sum can be evaluated directly for the full groupoid algebra

$$
A=\bigoplus_{x, y \in X} A_{x y}
$$

and so the vector space associated with the unit interval is precisely this direct sum. If one restricts it to the subspaces $A_{x y}$ corresponding to the boundary colours $x, y \in X$ of a given interval, one obtains an $X$-coloured open-closed TQFT. The full state sum with $A$, however, contains more than just these homogeneous elements. It includes their linear combinations as well. 
This last example is especially relevant in the context where the open-closed cobordisms are interpreted as open and closed string worldsheets. In this case, the colours of an $X$-coloured knowledgeable Frobenius algebra are interpreted as the set of boundary conditions, or D-branes, for the open strings. The decomposition of the finite groupoid algebra then allows the state sum to compute topological invariants of open and closed string worldsheets equipped with D-brane labels from the set of objects $X$ of the groupoid $\mathcal{G}$.

\section{Acknowledgments}

HP would like to thank Emmanuel College for a Research Fellowship. HP has performed a part of this work at Emmanuel College and at the Department of Applied Mathematics and Theoretical Physics (DAMTP), University of Cambridge, UK. Both authors would like to thank John Baez and Martin Hyland for helpful discussions and Ingo Runkel for correspondence. We thank Ezra Getzler for bringing the article [11] to our attention. Both authors are grateful to the European Union Superstring Theory Network for support.

\section{References}

[1] M. F. AтіYAн: Topological quantum field theories. IHES Publ. Math. 68 (1988) 175-186, MR 1001453

[2] R. Dijkgraff: A geometric approach to two dimensional conformal field theory. Ph.D. thesis, University of Utrecht, Utrecht (1989).

[3] L. Abrams: Two-dimensional topological quantum field theories and Frobenius algebras. $J$. Knot Th. Ramif. 5, No. 5 (1996) 569-587, MR 1414088

[4] S. F. SAwin: Direct sum decompositions and indecomposable TQFTs. J. Math. Phys. 36, No. 12 (1995) 6673-6680, MR 1359651, q-alg/9505026.

[5] J. Kock: Frobenius Algebras and 2D Topological Quantum Field Theories. London Mathematical Society Student Texts 59. Cambridge University Press, Cambridge, 2004. MR 2037238

[6] M. Fukuma, S. Hosono and H. Kawai: Lattice topological field theory in two dimensions. Comm. Math. Phys. 161, No. 1 (1994) 157-175, MR 1266073, hep-th/9212154.

[7] J. L. Cardy and D. C. Lewellen: Bulk and boundary operators in conformal field theory. Phys. Lett. B 259, No. 3 (1991) 274-278, MR 1107480

[8] D. C. Lewellen: Sewing constraints for conformal field theories on surfaces with boundaries. Nucl. Phys. B 372, No. 3 (1992) 654-682, MR 1156005

[9] C. I. Lazaroiu: On the structure of open-closed topological field theory in two dimensions. Nucl. Phys. B 603, No. 3 (2001) 497-530, MR 1839382 hep-th/0010269

[10] G. Moore and G. B. Segal: Lectures on Branes, $K$-theory and $R R$ Charges. Lecture notes from the Clay Institute School on Geometry and String Theory held at the Isaac Newton Institute, Cambridge, UK, available at http://www.physics.rutgers.edu/ gmoore/clay1/clay1.html and from the ITP Miniprogram 'The Duality Workshop' at Santa Barbara available at http://online.itp.ucsb.edu/online/mp01/moore1/ (2001-2002).

[11] A. Alexeevski and S. M. Natanzon: Noncommutative two-dimensional Topological Field Theories and Hurwitz numbers for real algebraic curves. To appear in Sel. Math. (2006). Preprint math.GT/0202164 
[12] A. D. Lauda and H. Pfeiffer: Open-closed strings: Two-dimensional extended TQFTs and Frobenius algebras (2005). Preprint math.AT/0510664

[13] J. Fuchs, I. Runkel and Ch. Schweigert: Conformal correlation functions, Frobenius algebras and triangulations. Nucl. Phys. B 624 (2002) 452-468, MR 1882479, hep-th/0110133

[14] I. Runkel, J. Fuelstad, J. Fuchs and Ch. Schweigert: Topological and conformal field theory as Frobenius algebras (2005). Preprint math.CT/0512076

[15] V. G. Turaev and O. Y. Viro: State sum invariants of 3-manifolds and quantum $6 j$ symbols. Topology 31, No. 4 (1992) 865-902, MR 1191386.

[16] J. W. BARrett and B. W. Westbury: Invariants of piecewise-linear 3-manifolds. Trans. Amer. Math. Soc. 348, No. 10 (1996) 3997-4022, MR 1357878, hep-th/9311155

[17] J. W. Barrett and B. W. Westbury: Spherical categories. Adv. Math. 143 (1999) 357-375, MR 1686423, hep-th/9310164

[18] J. Morton: 2d and 3d TQFTs and higher-dimensional algebra. Poster at the conference Loops '05, Potsdam, Germany. Available at http://math.ucr.edu/ morton/FHKposter.ps (2005).

[19] L. Crane and I. B. Frenkel: Four-dimensional topological quantum field theory, Hopf categories, and the canonical bases. J. Math. Phys. 35, No. 10 (1994) 5136-5154, MR 1295461. hep-th/9405183

[20] U. PAChNeR: PL homeomorphic manifolds are equivalent by elementary shellings. Europ. J. Combinat. 12 (1991) 129-145, MR 1095161

[21] L. Crane and D. N. Yetter: On algebraic structures implicit in topological quantum field theory. J. Knot Th. Ramif. 8, No. 2 (1999) 125-163, MR 1687553, hep-th/9412025

[22] D. N. Yetter: Portrait of the handle as a Hopf algebra. In Geometry and physics, eds. J. E. Andersen, J. Dupont et al., Lecture Notes in Pure and Applied Mathematics 184. Marcel Dekker, New York, 1997, pp. 481-502, MR 1423189

[23] T. KERLER: Towards an algebraic characterization of 3-dimensional cobordisms. In Diagrammatic morphisms and applications, eds. D. E. Radford, F. J. O. Souza and D. N. Yetter, Contemporary Mathematics 318. American Mathematical Society, Providence, 2003, pp. 141173, MR 1973515, math.GT/0106253

[24] G. Kuperberg: Involutory Hopf algebras and 3-manifold invariants. Int. J. Math. 2, No. 1 (1991) 41-66, MR 1082836, math.QA/9201301

[25] A. Joyal and R. Street: The geometry of tensor calculus. I. Adv. Math. 88, No. 1 (1991) $55-112$, MR 1113284

[26] R. Street: Low-dimensional topology and higher-order categories I. Canadian Mathematical Society Annual Seminar, Dalhousie University, Halifax, Canada, available at http://www . maths.mq.edu.au/ street/LowDTop.pdf (1995).

[27] S. MacLane: Natural associativity and commutativity. Rice Univ. Studies 49, No. 4 (1963) $28-46$, MR 0170925

[28] J. Fuchs and Ch. Schweigert: Category theory for conformal boundary conditions. Fields Inst. Comm. 39 (2003) 25-71, MR 2029790 math.CT/0106050

[29] M. Aguiar: A note on strongly separable algebras. Bol. Acad. Nac. Cienc. 65 (2000) 51-60, MR 1840439. 
[30] R. S. Pierce: Associative algebras. Graduate Texts in Mathematics 88. Springer, Berlin, 1982. MR 0674652

[31] F. Borceux: Handbook of categorical algebra - Basic category theory, volume 2. Cambridge University Press, Cambridge, 1994. MR 1313497.

[32] C. P. Rourke and B. J. Sanderson: Introduction to piecewise-linear topology. Ergebnisse der Mathematik und ihrer Grenzgebiete 69. Springer, Berlin, 1972. MR 0350744

[33] A. A. Ranicki (ed.): The Hauptvermutung Book. K-Monographs in Mathematics 1. Kluwer Academic Publishers, Dordrecht, 1996. MR 1434101

[34] N. A. BaAs, R. L. Cohen and A. Ramírez: The topology of the category of open and closed strings (2004). Preprint math.AT/0411080 